\title{
Side-bridged cyclam transition metal complexes bearing a phenolic ether or a phenolate pendent arm
}

\author{
Ryan E. Mewis ${ }^{*[a] \dagger}$ and Stephen J. Archibald $*[a, b, c]$
}

[a] Department of Chemistry and Biochemistry, University of Hull, Cottingham Road, Hull, HU6 7RX
[b] Department of Biomedical Sciences, University of Hull, Cottingham Road, Hull, HU6 7RX
[c] Positron Emission Tomography Research Centre, University of Hull, Cottingham Road, Hull, HU6 7RX
Fax: +44 (0) 1482466410
E-mail: s.j.archibald@ @ull.ac.uk
$\dagger \quad$ Current address: Department of Natural Sciences, Manchester Metropolitan University, John Dalton Building, Chester St., Manchester, M1 5GD, UK

\section{Abstract}

Side-bridged cyclam transition metal complexes $(\mathrm{M}=\mathrm{Ni}(\mathrm{II}), \mathrm{Cu}(\mathrm{II})$ and $\mathrm{Zn}(\mathrm{II}))$ bearing a phenolic ether or phenolate pendent arm have been synthesised. For $\left[\mathrm{NiL}^{1}\right]^{+}$and $\left[\mathrm{CuL}^{1}\right]^{+}$, evidence for a phenoxyl radical was obtained (quasi reversible peak at $+0.74 \mathrm{~V}$ and $+0.48 \mathrm{~V}$ respectively), as well as oxidation of $\mathrm{OH}$, due to protonation of the phenolate, at $\sim+1.24 \mathrm{~V}$. The phenoxyl radical in $\left[\mathrm{Ni}\left(\mathbf{L}^{1}\right)\right]^{2+}$ is harder to oxidise by $0.26 \mathrm{~V}$ compared with the corresponding $\mathrm{Cu}(\mathrm{II})$ complex. UV-Vis data for $\left[\mathrm{Ni}\left(\mathbf{L}^{1}\right)\right]^{2+}$ suggests that the $\mathrm{Ni}(\mathrm{II})$ ion may be 4 or 6 coordinate whereas the $\mathrm{Cu}(\mathrm{II})$ ion in $\left[\mathrm{Cu}\left(\mathbf{L}^{1}\right)\right]^{2+}$ is five coordinate. The $\mathrm{Ni}(\mathrm{II})$ ion in the crystal structure of $\left[\mathrm{Ni}\left(\mathbf{L}^{2}\right)\right]\left[\left(\mathrm{ClO}_{4}\right)_{2}\right]$ possesses a distorted squareplanar geometry in which the phenolic ether pendent arm is not involved in the coordination sphere. The cyclam ligand in this complex adopts a trans-II configuration.

Keywords: macrocyclic ligands / azamacrocycles / cyclam / bioinorganic / coordination chemistry

\section{Introduction}

Chelators based on tetraazamacrocycles, such as 1,4,8,11-tetraazacyclotetradecane (cyclam), have been utilised in medicine and biology for use as radiopharmaceuticals, ${ }^{1-4}$ anti-cancer drugs $^{5-7}$, magnetic resonance imaging (MRI) contrast agents, ${ }^{8,9}$ combined positron emission tomography (PET)/therapy theranostic compounds, ${ }^{10-14}$ and anti-HIV (Human Immunodeficiency Virus) drugs. ${ }^{15-20}$

Cyclam rings bearing pendent arms have been the focus of many research groups. ${ }^{1,16,21-24}$ The inclusion of pendent arms in to the ligand framework enables the resulting complex to be specifically engineered towards the desired application. ${ }^{25,26}$ The chemical design of the pendent arm can be used to modulate the coordination sphere surrounding the metal to increase stability. Additionally, they can be used to provide attachment points for biomolecules to increase the specificity of the complexes' in vivo activity ${ }^{1}$ or to act as chromophore ${ }^{27}$ to increase quantum yields of luminescence processes/ sensing applications. ${ }^{28,29}$ The ability of pendent arms to utilise more than one design feature is exemplified by 1,4,8,11-tetraazacyclotetradecane-1,4,8,11-tetraacetic acid (TETA), ${ }^{2}$ an extensively used bifunctional chelator (BFC) for copper radionuclides in clinical imaging and therapy studies involving both antibodies and peptides.

Incorporation of phenolate pendent arms to cyclam derived chelators is of interest as stabilised phenoxyl radical species can be generated. These radical species are important in a number of copper-containing proteins such as cyctochrome $\mathrm{c}$ oxidase, photosystem II and galactose oxidase. ${ }^{30,31}$ The complexed metal ion influences the ease of which the phenolate pendent arm can be oxidised and thus, selection of an appropriate metal ion can be used to tune the redox chemistry of the resulting metal complex..$^{32,33}$ 
Examples of cyclam ligands bearing phenolate pendent arms are known. Nirmala et al. ${ }^{34}$ have reported on the antimicrobial and electrochemical properties of $\mathrm{Cu}(\mathrm{II})$ and $\mathrm{Ni}(\mathrm{II})$ dioxocyclam complexes which have phenolate pendent arms. The inclusion of the metal into the macrocyclic cavity elevates antimicrobial and antifungal activity compared with the free ligand. A further example uses 2,4-dimethylphenol as a pendent arm to decorate the periphery of 1,4,7triazacyclonane to increase the hydrophobicity of the resulting gallium(III) complex in order to study its potential in targeted radiotherapy. ${ }^{35}$

Kimura and co-workers investigated the properties of a phenol containing cyclam ligand synthesised from coumarin. ${ }^{21,36-}$ 40 Several reports on this rigid phenol containing cyclam ligand and its transition metal complexes have been published. ${ }^{36,39,21}$ The X-ray structure of the copper(II) complex shows that the phenolic oxygen forms an axial interaction with the copper centre which adopts a five coordinate geometry. ${ }^{39}$ An unusually large thermal motion exists between some of the carbon atoms suggesting that the ligand has some flexibility on coordination. The nickel(II) complex shows a significantly lowered redox potential of $+0.35 \mathrm{~V}$ vs SCE $\left(0.5 \mathrm{M} \mathrm{Na}_{2} \mathrm{SO}_{4}, \mathrm{pH} 7.5,25^{\circ} \mathrm{C}\right)$ for $\mathrm{Ni}^{\mathrm{III} / \mathrm{II}}$ with respect to that of nickel(II)-cyclam $(+0.50 \mathrm{~V}$ vs. SCE), thus highlighting the potential of these complexes to tune metal redox properties. ${ }^{36}$ Conversely, the phenol moiety also becomes harder to oxidise upon coordination to nickel(II) ( $+0.5 \mathrm{~V}$ uncoordinated, $\sim+0.9 \mathrm{~V}$ coordinated). ${ }^{21}$

More recently, Maria et al. synthesised rare earth complexes of a dianionic cyclam-based ligand which possessed phenolic pendent arms. ${ }^{41,42}$ From X-ray crystallographic studies, the preference for the formation of either distorted octahedral or trigonal prismatic complexes was rationalised by considering the size of the rare earth ion.

Appending pendent arms to the cyclam backbone can be problematic due to the reactivity of the four nitrogen atoms. Consequently routes to circumvent this have been designed, ${ }^{43-45}$ such as the inclusion of a bridge by reaction with glyoxal to form the bisaminal. ${ }^{46-48}$ The cis-configuration of the central two-carbon bridge results in a folded geometry that governs nitrogen reactivity. Two nitrogen atoms have their lone pairs directed towards the convex side of the molecular structure whereas the other two nitrogens have their lone pairs directed towards the concave fold. Mono-alkylation is further favoured as the salt formed after nucleophilic attack precipitates from the reaction mixture. The bisaminal can be reduced in subsequent steps to give the side-bridged cyclam species, which is more rigid than its cyclam analogue and, therefore, affords the resulting metal complex increased kinetic stability. ${ }^{49,50}$

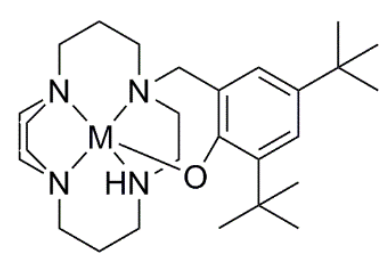

$\mathrm{ML}^{1}$

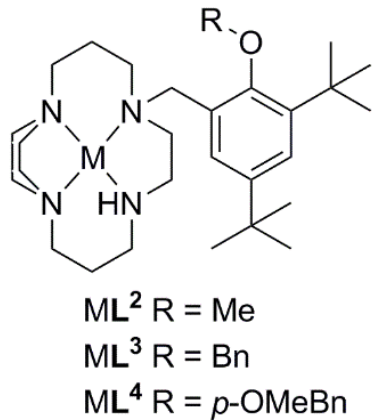

Figure 1. Structures of chelators and complexes discussed in this study (M= Ni(II), $\mathrm{Cu}$ (II) or $\mathrm{Zn}$ (II)).

In this paper we report on the design and synthesis of side-bridged cyclam ligands bearing phenolate $\left(\mathbf{L}^{\mathbf{1}}\right)$ or phenolic ether $\left(\mathbf{L}^{2}-\mathbf{L}^{\mathbf{4}}\right)$ pendent arms and their resulting $\mathrm{Ni}(\mathrm{II}), \mathrm{Cu}(\mathrm{II})$ and $\mathrm{Zn}(\mathrm{II})$ complexes, as shown in Figure 1 . Through the use of electrochemical and UV-Vis studies, the effect of the chelating ligand on the redox potentials of the $\mathrm{Cu}(\mathrm{II})$ and $\mathrm{Ni}(\mathrm{II})$ centres are probed and the ease of phenolate moiety oxidation for $\mathbf{L}^{\mathbf{1}}$ is investigated. 


\section{Experimental}

All chemicals and materials for synthetic procedures were purchased from Sigma Aldrich and used as received. The solvents used were of general purpose or HPLC grade and were purchased from Fisher Scientific. TLC analysis was performed using aluminium-backed silica gel $60 \mathrm{~F}_{254}, 0.2$ (Merck plates) or aluminium-backed aluminium oxide $60 \mathrm{~F}_{254}$, (Merck plates). Silica gel chromatography was performed with silica gel 60 (Davisil). When required diethyl ether, dichloromethane, acetonitrile and acetic acid were dried as follows; diethyl ether was dried over sodium metal and benzophenone followed by distillation; dichloromethane and acetonitrile were dried over calcium hydride for $24 \mathrm{~h}$ followed by distillation; acetic acid was dried by adding acetic anhydride ( $3 \% \mathrm{w} / \mathrm{v})$ and distilling (b.p. $\left.118^{\circ} \mathrm{C}\right)$.

NMR spectra were recorded using a JEOL JNM-LA400 FT NMR spectrometer at a frequency of $400 \mathrm{MHz}$ for ${ }^{1} \mathrm{H}$ spectra and $100 \mathrm{MHz}$ for ${ }^{13} \mathrm{C}$ spectra. Mass spectrometry was performed using a Finnegan MAT 900 XLT system to collect electrospray ionization (ES-MS). Accurate mass spectrometry measurements were obtained using a LTQ Orbitrap XL. UV-Visible spectra were obtained using an Agilent 8453E UV-VIS diode array spectrometer using $1 \mathrm{~cm}^{3}$ quartz cells.

Single crystal X-ray diffraction data were collected on a Stöe IPDS-II imaging plate diffractometer, using MoK $\alpha$ X-rays of $\lambda=0.71073 \AA$. Crystals were cooled to $150 \mathrm{~K}$ during data collection, with the temperature controlled by an Oxford Systems Cryostream Cooler. Diffraction data were solved using direct methods (SHELXS), and the refinement was by full-matrix least squares against $\mathrm{F}^{2}$ (SHELXL-97) ${ }^{51}$ method. The WinGX program ${ }^{52}$ was used for refinement and production of data tables, and the ORTEP-3 program $^{53}$ was used for structural visualisation. Hydrogen atoms were fixed in idealised positions and refined using a riding model, with $\mathrm{C}-\mathrm{H}$ distances of $0.97 \AA$, N-H distances of $0.91 \AA$, and $\mathrm{U}_{\text {iso }}$ 1.5 times $\mathrm{U}_{\text {eq }}$ of the carrier atom. All ORTEP representations show ellipsoids at the 50\% probability level.

Cyclic voltammetry was performed using a standard three-electrode configuration with platinum working $(0.2 \mathrm{~mm}$ diameter disk) and counter electrodes and a $\mathrm{Ag} / \mathrm{AgCl}$ reference which gave the $\mathrm{FeCp} / \mathrm{FeCp}^{+}$couple at $0.55 \mathrm{~V}$ using an Autolab II PGSTAT 30 system. All measurements were made in a MeCN argon/nitrogen purged solution containing the metal complex $(1 \mathrm{mM})$ and $\left[\mathrm{n}-\mathrm{Bu}_{4} \mathrm{~N}\right]\left[\mathrm{ClO}_{4}\right](0.2 \mathrm{M})$ over the scan rates of $0.01 \mathrm{~V} \mathrm{~s}^{-1}$ to $10 \mathrm{~V} \mathrm{~s}^{-1}$.

Synthesis of 3,5-di-tert-butyl-2-methoxybenzaldehyde (2)

$\mathrm{K}_{2} \mathrm{CO}_{3}(1.5 \mathrm{~g}, 0.011 \mathrm{~mol})$ was suspended in acetone $(30 \mathrm{ml})$ and stirred at $\mathrm{RT}$ (room temperature) for 5 min before adding 3,5-di-tert-butyl-2-hydroxybenzaldehyde $(0.5 \mathrm{~g}, 0.00214 \mathrm{~mol})$ and $\mathrm{MeI}(1.3 \mathrm{ml}, 0.0214 \mathrm{~mol})$. This yellow mixture was then stirred at RT for $15 \mathrm{hr}$, by which time the reaction was judged to be complete by TLC (5\% ethyl acetate in hexane). The solution was concentrated in vacuo and the white solid redissolved in hexane. The potassium carbonate was then removed by filtration and the filtrate concentrated to give 2 as a yellow oil $(0.44 \mathrm{~g}, 83 \%)$ : $\mathrm{R}_{\mathrm{f}}=0.5$ (5\% ethyl acetate in hexane); ${ }^{1} \mathrm{H}$ NMR (400 MHz, $\left.\mathrm{CDCl}_{3}\right) \delta 10.22(\mathrm{~s}, 1 \mathrm{H}, \mathrm{CHO}), 7.61(\mathrm{~d}, 1 \mathrm{H}, J=2.5 \mathrm{~Hz}, \mathrm{ArH}), 7.51(\mathrm{~d}, 1 \mathrm{H}, J=2.5 \mathrm{~Hz}, \mathrm{ArH})$, $3.82(\mathrm{~s}, 3 \mathrm{H}, \mathrm{OMe}), 1.32\left(\mathrm{~s}, 9 \mathrm{H},{ }^{\mathrm{t}} \mathrm{Bu}\right), 1.22\left(\mathrm{~s}, 9 \mathrm{H},{ }^{\mathrm{t}} \mathrm{Bu}\right) ;{ }^{13} \mathrm{C} \mathrm{NMR}\left(100 \mathrm{MHz}, \mathrm{CDCl}_{3} 0 \delta 190.7\right.$ (CHO), 166.2, 146.2, 142.8 , 130.6, 129.0, $124.2\left(\mathrm{C}_{\text {arom }}\right), 65.9(\mathrm{OMe}), 35.2,34.6\left(\mathrm{C}\left({ }^{\mathrm{t}} \mathrm{Bu}\right)\right), 31.2,30.8\left(\mathrm{CH}_{3}\left({ }^{\mathrm{t}} \mathrm{Bu}\right)\right)$; MS (ES-MS): $\mathrm{m} / 2.248\left(\mathrm{M}^{+}\right)$.

Synthesis of (3,5-di-tert-butyl-2-methoxyphenyl) methanol (5)

$2(0.44 \mathrm{~g}, 1.77 \mathrm{mmol})$ was dissolved in ethanol $(30 \mathrm{ml})$ to which was added $\mathrm{NaBH}_{4}(0.1 \mathrm{~g}, 2.63 \mathrm{mmol})$ portionwise. This yellow solution was then stirred at RT for $2.5 \mathrm{hr}$ before concentrating under reduced pressure prior to partitioning between $\mathrm{CH}_{2} \mathrm{Cl}_{2}(60 \mathrm{ml})$ and brine $(200 \mathrm{ml})$. The organic layer was collected and dried over $\mathrm{MgSO}_{4}$ before removing the solvent in vacuo to give a yellow oil. This was placed in a fridge overnight whereby the oil solidified to give a white solid (0.44 g, 100\%); ${ }^{1} \mathrm{H}$ NMR (400 MHz, $\left.\mathrm{CDCl}_{3}\right) \delta 7.30$ (d, 1H, J=2.5 Hz, ArH), 7.29 (d, 1H, J=2.5 Hz, ArH), 5.26 (s, $1 \mathrm{H}, \mathrm{OH}), 4.74\left(\mathrm{~s}, 2 \mathrm{H}, \mathrm{CH}_{2}\right), 3.78(\mathrm{~s}, 3 \mathrm{H}, \mathrm{OMe}), 1.40\left(\mathrm{~s}, 9 \mathrm{H},{ }^{\mathrm{t}} \mathrm{Bu}\right), 1.31\left(\mathrm{~s}, 9 \mathrm{H},{ }^{\mathrm{t}} \mathrm{Bu}\right) ;{ }^{13} \mathrm{C} \mathrm{NMR}\left(100 \mathrm{MHz}, \mathrm{CDCl}_{3}\right) \delta$ 
155.3, 146.0, 141.8, 133.4, 124.4, $123.9\left(\mathrm{C}_{\text {arom }}\right), 61.9(\mathrm{OMe}), 61.8\left(\mathrm{CH}_{2} \mathrm{OH}\right), 35.3,34.5\left(\mathrm{C}\left({ }^{\mathrm{t}} \mathrm{Bu}\right)\right), 31.5,31.1\left(\mathrm{CH}_{3}\left({ }^{\mathrm{t}} \mathrm{Bu}\right)\right)$; MS (ES-MS): $m / z 250\left(\mathrm{M}^{+}\right)$

Synthesis of 1-(bromomethyl)-3,5-di-tert-butyl-2-methoxybenzene (8)

$5(0.44 \mathrm{~g}, 1.41 \mathrm{mmol})$ was dissolved in $\mathrm{CHCl}_{3}(25 \mathrm{ml})$ at $0^{\circ} \mathrm{C} . \mathrm{PBr}_{3}(0.38 \mathrm{~g}, 1.41 \mathrm{mmol})$ dissolved in $\mathrm{CHCl}_{3}(15 \mathrm{ml})$ was then added dropwise over 30 min under a nitrogen atmosphere. The yellow solution was then stirred at $0^{\circ} \mathrm{C}$ for a further $1 \mathrm{hr}$ before the organic layer was washed with brine $(3 \times 200 \mathrm{ml})$. The organic layer was collected, dried over $\mathrm{Na}_{2} \mathrm{SO}_{4}$ and concentrated in vacuo to give 8 as a yellow oil $(0.47,85 \%) ;{ }^{1} \mathrm{H} \mathrm{NMR}\left(400 \mathrm{MHz}, \mathrm{CDCl}_{3}\right) \delta 7.36(\mathrm{~d}, 1 \mathrm{H}, J=2.5 \mathrm{~Hz}$, $\operatorname{ArH}), 7.34$ (d, 1H, J=2.5 Hz, ArH), 4.63 (s, 2H, $\left.\mathrm{CH}_{2}\right), 3.90(\mathrm{~s}, 3 \mathrm{H}, \mathrm{OMe}), 1.45\left(\mathrm{~s}, 9 \mathrm{H},{ }^{\mathrm{t}} \mathrm{Bu}\right), 1.36\left(\mathrm{~s}, 9 \mathrm{H},{ }^{\mathrm{t}} \mathrm{Bu}\right) ;{ }^{13} \mathrm{C} \mathrm{NMR}$ $\left(100 \mathrm{MHz}, \mathrm{CDCl}_{3}\right) \delta 155.7,146.3,142.3,130.7,127.1,125.1\left(\mathrm{C}_{\text {arom }}\right), 62.2(\mathrm{OMe}), 35.4,34.5\left(\mathrm{C}\left({ }^{\mathrm{t}} \mathrm{Bu}\right)\right), 31.4,31.1$ $\left(\mathrm{CH}_{3}\left({ }^{\mathrm{t}} \mathrm{Bu}\right)\right), 23.0\left(\mathrm{CH}_{2} \mathrm{Br}\right)$; MS (ES-MS): $m / z 312\left([\mathrm{M}-\mathrm{H}]^{+}\right), 233\left([\mathrm{M}-\mathrm{Br}]^{+}\right)$.

Synthesis of 2-(benzyloxy)-3,5-di-tert-butylbenzaldehyde (3)

3,5-Di-tert-butyl-2-hydroxybenzaldehyde $(4.0 \mathrm{~g}, 0.0171 \mathrm{~mol})$ and potassium carbonate $(2.1 \mathrm{~g}, 0.0171 \mathrm{~mol})$ were dissolved in DMF $(25 \mathrm{ml})$ at $0^{\circ} \mathrm{C}$ and stirred for $5 \mathrm{~min}$. To the yellow solution was added benzyl bromide $(2.89 \mathrm{~g}, 0.0168$ $\mathrm{mol}$ ) and the resulting mixture was heated to $75^{\circ} \mathrm{C}$ for $24 \mathrm{hr}$ before cooling and pouring into ice-water (200 $\left.\mathrm{ml}\right)$. The precipitate formed by this process was then collected by filtration and washed with water $(2 \times 10 \mathrm{ml})$ to give 3 as fine crystals $(5.0 \mathrm{~g}, 90 \%) ;{ }^{1} \mathrm{H}$ NMR $\left(400 \mathrm{MHz}, \mathrm{CDCl}_{3}\right) \delta 10.34(\mathrm{~s}, 1 \mathrm{H}, \mathrm{CHO}), 7.75(\mathrm{~d}, 1 \mathrm{H}, J=2.5 \mathrm{~Hz}, \mathrm{ArH}), 7.66(\mathrm{~d}, 1 \mathrm{H}$, $J=2.5 \mathrm{~Hz}, \mathrm{ArH}), 7.51-7.36(\mathrm{~m}, 5 \mathrm{H}, \mathrm{Bn}), 5.04\left(\mathrm{~s}, 2 \mathrm{H}, \mathrm{CH}_{2}\right), 1.46\left(\mathrm{~s}, 9 \mathrm{H},{ }^{\mathrm{t}} \mathrm{Bu}\right), 1.34\left(\mathrm{~s}, 9 \mathrm{H},{ }^{\mathrm{t}} \mathrm{Bu}\right) ;{ }^{13} \mathrm{C} \mathrm{NMR}(100 \mathrm{MHz}$, $\left.\mathrm{CDCl}_{3}\right) \delta 190.8(\mathrm{CHO}), 159.7,146.6,143.1,136.6,131.0,129.3,128.6,128.1,127.0,124.0\left(\mathrm{C}_{\text {arom }}\right), 80.4\left(\mathrm{CH}_{2}\right), 35.4$, $34.7\left(\mathrm{C}\left({ }^{\mathrm{t}} \mathrm{Bu}\right)\right), 31.3,30.9\left(\mathrm{Me}\left({ }^{\mathrm{t}} \mathrm{Bu}\right)\right)$; $\mathrm{MS}(\mathrm{ES}-\mathrm{MS}): \mathrm{m} / z 354\left(\mathrm{M}^{+}\right)$.

Synthesis of (2-(benzyloxy)-3,5-di-tert-butylphenyl)methanol (6)

To a solution of 3 (4.50 g, $0.0139 \mathrm{~mol})$ dissolved in methanol $(50 \mathrm{ml})$ was added $\mathrm{NaBH}_{4}(1.05 \mathrm{~g}, 0.0278 \mathrm{~mol})$ portion wise over 5 min. After addition was complete, the yellow solution was stirred at RT for an hour before removing the solvent in vacuo. The white solid formed was then partitioned between $\mathrm{CHCl}_{3}(50 \mathrm{ml})$ and brine $(200 \mathrm{ml})$. The organic layer was collected and further washed with brine $(2 \times 200 \mathrm{ml})$. The organic extracts were then combined, dried over $\mathrm{MgSO}_{4}$ and concentrated in vacuo to give 6 as a pale yellow oil $(4.50 \mathrm{~g}, 99 \%) ;{ }^{1} \mathrm{H} \mathrm{NMR}\left(400 \mathrm{MHz}, \mathrm{CDCl}_{3}\right) \delta 7.50-7.30$ (m, 7H, ArH), 4.97 (s, 2H, $\left.\mathrm{CH}_{2}, \mathrm{CH}_{2} \mathrm{Bn}\right), 4.74$ (s, 2H, $\left.\mathrm{CH}_{2} \mathrm{OH}\right), 1.43$ (s, 9H, $\left.{ }^{\mathrm{t}} \mathrm{Bu}\right), 1.32\left(\mathrm{~s}, 9 \mathrm{H},{ }^{\mathrm{t}} \mathrm{Bu}\right) ;{ }^{13} \mathrm{C} \mathrm{NMR}(100 \mathrm{MHz}$, $\left.\mathrm{CDCl}_{3}\right) \delta 153.8,146.2,142.1,137.6,133.7,128.5,127.6,126.8,124.8,124.1\left(\mathrm{C}_{\text {arom }}\right), 75.8\left(\mathrm{CH}_{2} \mathrm{Bn}\right), 61.5\left(\mathrm{CH}_{2} \mathrm{OH}\right)$, 35.4, $34.5\left(\mathrm{C}\left({ }^{\mathrm{t}} \mathrm{Bu}\right)\right), 31.5,31.2\left(\mathrm{Me}\left({ }^{\mathrm{t}} \mathrm{Bu}\right)\right)$; $\mathrm{MS}(\mathrm{ES}-\mathrm{MS}): \mathrm{m} / z .356\left(\mathrm{M}^{+}\right)$.

Synthesis of 2-(benzyloxy)-1-(bromomethyl)-3,5-di-tert-butylbenzene (9)

To $6(2.07 \mathrm{~g}, 0.00635 \mathrm{~mol})$ in $\mathrm{CHCl}_{3}(50 \mathrm{ml})$ at $0^{\circ} \mathrm{C}$ under nitrogen was added $\mathrm{PBr}_{3}(1.72 \mathrm{~g}, 1.5 \mathrm{ml}, 0.00635 \mathrm{~mol})$ in $\mathrm{CHCl}_{3}(30 \mathrm{ml})$ over $30 \mathrm{~min}$. The solution was then stirred at $0^{\circ} \mathrm{C}$ for $1 \mathrm{hr}$ before pouring into brine $(200 \mathrm{ml})$. The organic layer was extracted and then washed further with brine $(3 \times 200 \mathrm{ml})$. The organic extracts were combined, dried over $\mathrm{Na}_{2} \mathrm{SO}_{4}$ and then concentrated in vacuo to give 9 as a white solid $(2.36 \mathrm{~g}, 96 \%) ;{ }^{1} \mathrm{H} \mathrm{NMR}\left(400 \mathrm{MHz}, \mathrm{CDCl}_{3}\right) \delta 7.56-$ 7.34 (m, 7H, ArH), 5.12 (s, 2H, $\left.\mathrm{CH}_{2} \mathrm{Bn}\right), 4.58$ (s, 2H, $\left.\mathrm{CH}_{2} \mathrm{Br}\right), 1.43$ (s, 9H, $\left.{ }^{\mathrm{t}} \mathrm{Bu}\right), 1.32$ (s, 9H, $\left.{ }^{\mathrm{t}} \mathrm{Bu}\right) ;{ }^{13} \mathrm{C} \mathrm{NMR}(100 \mathrm{MHz}$, $\left.\mathrm{CDCl}_{3}\right) \delta 154.2,146.5,142.5,137.6,130.9,128.5,127.7,127.3,126.8,125.3\left(\mathrm{C}_{\text {arom }}\right), 75.2\left(\mathrm{CH}_{2} \mathrm{Bn}\right), 35.6,34.5\left(\mathrm{C}\left({ }^{\mathrm{t}} \mathrm{Bu}\right)\right)$, 31.4, $31.2\left(\mathrm{Me}\left({ }^{\mathrm{t}} \mathrm{Bu}\right)\right), 30.1\left(\mathrm{CH}_{2} \mathrm{Br}\right)$; MS (ES-MS): $\mathrm{m} / z 388\left(\mathrm{M}^{+}\right)$; Calcd for $\mathrm{C}_{14} \mathrm{H}_{27} \mathrm{Br}_{1} \mathrm{O}_{1}: \mathrm{C}, 67.90$; H, 7.40. Found: C, $68.03 ; \mathrm{H}, 7.63$.

Synthesis of 3,5-di-tert-butyl-2-(4-methoxybenzyloxy)benzaldehyde (4) 
3,5-Di-tert-butyl-2-hydroxy benzaldehyde $(2 \mathrm{~g}, 0.0085 \mathrm{~mol})$ and $\mathrm{K}_{2} \mathrm{CO}_{3}(1.18 \mathrm{~g}, 0.0085 \mathrm{~mol})$ in $\mathrm{DMF}(40 \mathrm{ml})$ were stirred together for $5 \mathrm{~min}$. 1-(Bromomethyl)-4-methoxybenzene $(1.89 \mathrm{~g}, 0.0094 \mathrm{~mol})$ was then added and the solution heated to $55^{\circ} \mathrm{C}$ for $24 \mathrm{hr}$. The reaction mixture was allowed to cool before pouring in to ice-water $(60 \mathrm{ml})$. A precipitate formed which was removed by filtration. The crude solid was then subjected to hot pentane washes $(4 \times 10 \mathrm{ml})$. The resulting white solid was then recrystallised from hexane. This gave 4 as colourless crystals $(2.11 \mathrm{~g}, 70 \%) ;{ }^{1} \mathrm{H}$ NMR $\left(400 \mathrm{MHz}, \mathrm{CDCl}_{3}\right) \delta 10.35(\mathrm{CHO}), 7.75(\mathrm{~d}, 1 \mathrm{H}, J=2.67 \mathrm{~Hz}, \mathrm{ArH}), 7.67(\mathrm{~d}, 1 \mathrm{H}, J=2.67 \mathrm{~Hz}, \mathrm{ArH}), 7.43(\mathrm{~d}, 2 \mathrm{H}, J=8.7$, $\mathrm{ArH}), 6.95$ (d, $2 \mathrm{H}, J=8.7, \mathrm{ArH}), 4.96$ (s, 2H, CH $\mathrm{CH}_{2} \mathrm{Bn}$ ), 3.83 (s, 3H, OMe), 1.46 (s, 9H, $\left.{ }^{\mathrm{t}} \mathrm{Bu}\right), 1.34\left(\mathrm{~s}, 9 \mathrm{H},{ }^{\mathrm{t}} \mathrm{Bu}\right) ;{ }^{13} \mathrm{C}$ NMR $\left(100 \mathrm{MHz}, \mathrm{CDCl}_{3}\right) \delta 190.8$ (CHO), 159.7, 159.5, 146.4, 143.0, 130.9, 129.3, 128.7, 128.6, 123.9, $114.0\left(\mathrm{C}_{\text {arom }}\right), 80.3$ $\left(\mathrm{CH}_{2} \mathrm{Bn}\right), 55.2(\mathrm{OMe}), 35.3,34.7\left(\mathrm{C}\left({ }^{\mathrm{B}} \mathrm{Bu}\right)\right), 31.3,30.9\left(\mathrm{Me}\left({ }^{\mathrm{t}} \mathrm{Bu}\right)\right)$; MS (ES-MS): $m / 2354\left(\mathrm{M}^{+}\right)$.

Synthesis of (3,5-di-tert-butyl-2-(4-methoxybenzyloxy)phenyl)methanol (7)

To a solution of $4(1.5 \mathrm{~g}, 0.0042 \mathrm{~mol})$ in ethanol $(40 \mathrm{ml})$ was added $\mathrm{NaBH}_{4}(0.16 \mathrm{~g}, 0.0042 \mathrm{~mol})$ portionwise with stirring. The solution was stirred for 3 hours at RT before concentrating the mixture under reduced pressure. The residue was then partitioned between $\mathrm{CH}_{2} \mathrm{Cl}_{2}(100 \mathrm{ml})$ and water $(100 \mathrm{ml})$. The organic layer was collected, dried over $\mathrm{MgSO}_{4}$ and concentrated under reduced pressure. This gave 7 as a white solid $(1.21 \mathrm{~g}, 81 \%) ;{ }^{1} \mathrm{H} \mathrm{NMR}\left(400 \mathrm{MHz}, \mathrm{CDCl}_{3}\right) \delta 7.45(\mathrm{~d}$, 2H, J=8.4 Hz, ArH), 7.37 (d, 1H, J=2.5 Hz, ArH), 7.32 (d, 1H, J=2.53 Hz, ArH), 6.95 (d, 2H, J=8.4 Hz, ArH), 4.91 (s, $\left.2 \mathrm{H}, \mathrm{CH}_{2} \mathrm{Bn}\right), 4.79$ (s, 2H, $\left.\mathrm{CH}_{2} \mathrm{OH}\right), 3.82$ (s, 3H, OMe), 1.45 (s, 9H, $\left.{ }^{\mathrm{B}} \mathrm{Bu}\right), 1.34\left(\mathrm{~s}, 9 \mathrm{H},{ }^{\mathrm{t}} \mathrm{Bu}\right) ;{ }^{13} \mathrm{C} \mathrm{NMR}\left(400 \mathrm{MHz}, \mathrm{CDCl}_{3}\right)$ $\delta$ 159.2, 153.9, 146.2, 142.1, 133.7, 129.7, 128.6, 124.7, 124.2, $113.9\left(\mathrm{C}_{\text {arom }}\right), 75.7\left(\mathrm{CH}_{2} \mathrm{Bn}\right), 61.8\left(\mathrm{CH}_{2} \mathrm{OH}\right), 55.2(\mathrm{OMe})$, 35.4, 34.5, $\left(\mathrm{C}\left({ }^{(} \mathrm{Bu}\right)\right), 31.5,31.3$, $\left(\mathrm{Me}\left({ }^{\mathrm{t}} \mathrm{Bu}\right)\right)$; MS (ES-MS): $\mathrm{m} / \mathrm{z} 356\left(\mathrm{M}^{+}\right)$; Calcd for $\mathrm{C}_{23} \mathrm{O}_{3} \mathrm{H}_{32}: \mathrm{C}, 77.53 ; \mathrm{H}$ 8.99. Found $\mathrm{C}$, 77.54; H, 8.73 .

Synthesis of 1-(bromomethyl)-3,5-di-tert-butyl-2-(4-methoxybenzyloxy)benzene (10)

$7(1 \mathrm{~g}, 0.00281 \mathrm{~mol})$ and $\mathrm{CBr}_{4}(1.4 \mathrm{~g}, 0.00421 \mathrm{~mol})$ were dissolved in dry $\mathrm{CH}_{2} \mathrm{Cl}_{2}(20 \mathrm{ml})$ to which was added triphenylphosphine $(1.11 \mathrm{~g}, 0.00421 \mathrm{~mol})$ in dry $\mathrm{CH}_{2} \mathrm{Cl}_{2}(10 \mathrm{ml})$ over $5 \mathrm{~min}$. A yellow solution formed which was then stirred at RT for $18 \mathrm{hr}$. Water $(150 \mathrm{ml})$ was then added and the organic layer was extracted. The aqueous layer was further extracted with $\mathrm{CH}_{2} \mathrm{Cl}_{2}(2 \times 50 \mathrm{ml})$. All the organic extracts were combined and dried over $\mathrm{MgSO}_{4}$. The crude product was then purified by silica gel chromatography $\left(1: 1 \mathrm{CH}_{2} \mathrm{Cl}_{2} / \mathrm{hexane} \mathrm{v} / \mathrm{v}\right)$. The fractions containing the desired compound were then combined and concentrated under reduced pressure to give a light pink solid $(0.80 \mathrm{~g}, 68 \%) ; \mathrm{R}_{\mathrm{f}}=0.58$ (1:1 hexane/ $\left.\mathrm{CH}_{2} \mathrm{Cl}_{2} \mathrm{v} / \mathrm{v}\right) ;{ }^{1} \mathrm{H}$ NMR $\left(400 \mathrm{MHz}, \mathrm{CDCl}_{3}\right) \delta 7.52(\mathrm{~d}, 2 \mathrm{H}, J=8.6 \mathrm{~Hz}, \mathrm{ArH}), 7.38(\mathrm{~s}, 2 \mathrm{H}, \mathrm{ArH}), 6.89(\mathrm{~d}, 2 \mathrm{H}$, $J=8.6 \mathrm{~Hz}, \mathrm{ArH}), 5.18$ (s, 2H, $\mathrm{CH}_{2} \mathrm{Bn}$ ), 4.63 (s, 2H, $\mathrm{CH}_{2} \mathrm{Br}$ ), 3.86 (s, 3H, OMe), 1.47 (s, 9H, 'Bu), 1.36 (s, 9H, 'Bu); ${ }^{13} \mathrm{C}$ NMR (400 MHz, $\left.\mathrm{CDCl}_{3}\right) \delta 159.3,154.2,146.4,142.5,130.9,129.8,128.5,127.3,126.0,114.0\left(\mathrm{C}_{\text {arom }}\right), 75.1\left(\mathrm{CH}_{2} \mathrm{Bn}\right)$, $55.3(\mathrm{OMe}), 35.6,34.5\left(\mathrm{C}\left({ }^{ } \mathrm{Bu}\right)\right), 31.6\left(\mathrm{CH}_{2} \mathrm{Br}\right), 31.4,31.3\left(\mathrm{Me}\left({ }^{\mathrm{t}} \mathrm{Bu}\right)\right)$; MS (ES-MS) m/z $339\left((\mathrm{M}-\mathrm{Br})^{+}\right), 219([\mathrm{MH}-$ $\left.\left.\mathrm{C}_{8} \mathrm{H}_{9} \mathrm{O}_{1} \mathrm{Br}\right]^{+}\right)$.

Synthesis of 2-(bromomethyl)-4,6-di-tert-butylphenyl acetate (11)

To solution of 1 ( $3.0 \mathrm{~g}, 0.0100 \mathrm{~mol})$ in acetic anhydride $(2.05 \mathrm{~g}, 0.0201 \mathrm{~mol})$ was added $18 \mathrm{M} \mathrm{H}_{2} \mathrm{SO}_{4}$ (5 drops). The reaction mixture was stirred for $12 \mathrm{hr}$ at which time the TLC showed complete consumption of the starting material (silica plate using 5\% EtOAc in hexane as the eluent). $\mathrm{CH}_{2} \mathrm{Cl}_{2}(50 \mathrm{ml})$ was then added and the mixture washed with brine $(2 \times 100 \mathrm{ml})$. The organic layer was collected and dried over $\mathrm{MgSO}_{4}$ before filtering under reduced pressure. The resulting yellow oil was placed in a freezer whereby a solid formed overnight $(3.41 \mathrm{~g}, 100 \%) ;{ }^{1} \mathrm{H} \mathrm{NMR}(400 \mathrm{MHz}$, $\left.\mathrm{CDCl}_{3}\right) \delta 7.42$ (s, 1H, ArH), 7.32 (s, $\left.1 \mathrm{H}, \mathrm{ArH}\right), 4.34$ (s, 2H, $\mathrm{CH}_{2} \mathrm{Br}$ ), 2.42 (s, 3H, COMe), 1.37 (s, 9H, 'Bu), 1.33 (s, 9H, $\left.{ }^{\mathrm{t}} \mathrm{Bu}\right) ;{ }^{13} \mathrm{C}$ NMR $\left(100 \mathrm{MHz}, \mathrm{CDCl}_{3}\right) \delta 169.8$ (COMe), 148.7, 145.5, 141.4, 130.1, 126.4, $125.6\left(\mathrm{C}_{\text {arom }}\right), 35.1,34.8\left(\mathrm{C}\left({ }^{\mathrm{t}} \mathrm{Bu}\right)\right)$, 31.5, $30.7\left(\mathrm{Me}\left({ }^{\mathrm{t}} \mathrm{Bu}\right)\right), 29.7\left(\mathrm{CH}_{2} \mathrm{Br}\right), 21.6\left(\mathrm{COCH}_{3}\right)$; Calcd. for $\mathrm{C}_{17} \mathrm{O}_{2} \mathrm{Br}_{1} \mathrm{H}_{25}: \mathrm{C}, 59.82 ; \mathrm{H}, 7.33$. Found: $\mathrm{C}, 60.11 ; \mathrm{H}, 7.52$; 
MS (ES-MS): $m / z 261\left([\mathrm{M}-\mathrm{Br}]^{+}\right), 219.2\left(\left[\mathrm{MH}-\mathrm{CH}_{3} \mathrm{OBr}\right]^{+}\right)$.

Synthesis of N-3,5-di-tert-butyl-2-methoxy-benzyl-cis-3a,5a,8a,10a-tetraazaperhydropyrene bromide (14)

$12(0.21 \mathrm{~g}, 0.959 \mathrm{mmol})$ and $8(0.3 \mathrm{~g}, 0.958 \mathrm{mmol})$ were dissolved in dry MeCN $(20 \mathrm{ml})$ and stirred at RT for $5 \mathrm{days}$. The solvent was removed in vacuo and the residue washed with diethyl ether $(30 \mathrm{ml})$. A precipitate formed which was collected by filtration and washed with further ether $(3 \times 10 \mathrm{ml})$. This gave the product as a white solid $(0.33 \mathrm{~g}, 64 \%)$; ${ }^{1} \mathrm{H}$ NMR (400 MHz, CD 3 OD) $\delta 7.52$ (d, 1H, J=2.2 Hz, ArH), 7.33 (d, 1H, J=2.2 Hz, ArH), 4.76 (s, 5H, CH ), 4.21-4.15 $\left(\mathrm{m}, 2 \mathrm{H}, \mathrm{CH}_{2}\right), 3.72-3.49\left(\mathrm{~m}, 1 \mathrm{H}, \mathrm{CH}_{2}\right), 3.40-3.33\left(\mathrm{~m}, 1 \mathrm{H}, \mathrm{CH}_{2}\right), 3.21-2.87\left(\mathrm{~m}, 12 \mathrm{H}, \mathrm{CH}_{2}\right.$ and $\left.\mathrm{OMe}\right), 2.55-2.15(\mathrm{~m}, 6 \mathrm{H}$, $\left.\mathrm{CH}_{2}\right), 1.33$ (s, 9H, $\left.{ }^{\mathrm{B}} \mathrm{Bu}\right), 1.24\left(\mathrm{~s}, 9 \mathrm{H},{ }^{\mathrm{t}} \mathrm{Bu}\right) ;{ }^{13} \mathrm{C} \mathrm{NMR}\left(100 \mathrm{MHz}, \mathrm{CD}_{3} \mathrm{OD}\right) \delta 155.1,143.8,140.6,126.7,124.9,117.1\left(\mathrm{C}_{\text {arom }}\right)$, 80.1, $73.1(\underline{\mathrm{NCHN}}), 67.3(\underline{\mathrm{OMe}}), 60.0\left(\mathrm{NCH}_{2} \mathrm{Ph}\right), 55.9,55.6,51.5,50.9,49.1,48.9,45.9,39.3\left(\mathrm{CH}_{2}\right), 32.0,31.0\left(\mathrm{C}\left({ }^{\mathrm{t}} \mathrm{Bu}\right)\right)$, 27.2, $27.1\left(\mathrm{Me}\left({ }^{\mathrm{t}} \mathrm{Bu}\right)\right), 15.7,15.6\left(\mathrm{NCH}_{2} \underline{\mathrm{CH}}_{2} \mathrm{CH}_{2} \mathrm{~N}\right)$; MS (ES-MS): $\mathrm{m} / z 455.7\left(\mathrm{M}^{+}\right)$.

Synthesis of N-3,5-di-tert-butyl-2-benzylether-benzyl-cis 3a,5a,8a,10a-tetraazaperhydropyrene (15)

$12(0.45 \mathrm{~g}, 0.002 \mathrm{mmol})$ and $9(0.78 \mathrm{~g}, 0.002 \mathrm{mmol})$ were dissolved in MeCN (30 ml) and stirred at RT for $3 \mathrm{days}$. The MeCN was then removed in vacuo and the isolated oil was triturated diethyl ether (30 ml) to yield a white solid. This white solid was then filtered off and further washed with ether $(2 \times 30 \mathrm{ml})$ to give $\mathbf{3 8}$ as a white solid $(0.38 \mathrm{~g}, 66 \%) ;{ }^{1} \mathrm{H}$ NMR (400 MHz, CD $\left.{ }_{3} \mathrm{OD}\right) \delta$ 7.67-7.35 (m, 7H, ArH), 4.13-4.05 (m, 1H, $\left.\mathrm{CH}_{2}\right), 3.59-2.80\left(\mathrm{~m}, 12 \mathrm{H}, \mathrm{CH}_{2}\right), 3.04-2.80(\mathrm{~m}$, $\left.9 \mathrm{H}, \mathrm{CH}_{2}\right), 2.52-2.03\left(\mathrm{~m}, 4 \mathrm{H}, \mathrm{CH}_{2}\right), 1.34\left(\mathrm{~s}, 9 \mathrm{H},{ }^{\mathrm{t}} \mathrm{Bu}\right), 1.26\left(\mathrm{~s}, 9 \mathrm{H},{ }^{\mathrm{t}} \mathrm{Bu}\right) ;{ }^{13} \mathrm{C} \mathrm{NMR}\left(100 \mathrm{MHz}, \mathrm{CD}_{3} \mathrm{OD}\right) \delta 153.2,143.9$, 140.4, 133.4, 126.9, 125.3, 124.9, 124.8, 123.9, $117.0\left(\mathrm{C}_{\text {arom }}\right), 80.3,74.7(\mathrm{NCHN}), 72.9\left(\mathrm{OCH}_{2} \mathrm{Ph}\right), 66.9\left(\mathrm{NCH}_{2} \mathrm{Ph}\right), 56.1$, $55.9,50.8,50.7,49.0,48.4,39.0\left(\mathrm{CH}_{2}\right), 32.0,30.9\left(\mathrm{C}\left({ }^{\mathrm{t}} \mathrm{Bu}\right)\right), 27.3,27.0\left(\mathrm{Me}\left({ }^{\mathrm{t}} \mathrm{Bu}\right)\right), 15.5,15.2\left(\mathrm{NCH}_{2} \underline{\mathrm{CH}}_{2} \mathrm{CH}_{2} \mathrm{~N}\right)$; $\mathrm{MS}(\mathrm{ES}-$ MS): $m / z 532.4\left(\mathrm{M}^{+}\right)$.

Synthesis of 3a-3,5-di-tert-butyl-2-(4-methoxy-benzyloxy)-benzyl-decahyro-5a, 8a, 10a-triaaza-3a-azonia-pyrene bromide (16)

$12(0.53 \mathrm{~g}, 2.4 \mathrm{mmol})$ and $\mathbf{1 0}(1 \mathrm{~g}, 2.4 \mathrm{mmol})$ were dissolved in dry MeCN (20 ml) and stirred for 4 days at RT. After this time, a precipitate had formed. The reaction mixture was then concentrated under reduced pressure and filtered. The solid was then washed with pentane $(3 \times 20 \mathrm{ml})$, and the product was isolated as an orange solid $(1.35 \mathrm{~g}, 88 \%) ;{ }^{1} \mathrm{H} \mathrm{NMR}$ (400 MHz, CD 3 OD) $\delta 7.57$ (d, 1H, J=2.4 Hz, ArH), 7.42 (d, 2H, J=8.6 Hz, ArH), 7.39 (d, 1H, J=2.4 Hz, ArH), 6.94 (d, $2 \mathrm{H}, J=8.6 \mathrm{~Hz}, \mathrm{ArH}), 4.78$ (s, 2H, $\left.\underline{\mathrm{NCH}}_{2} \mathrm{Phen}\right) ; 3.73$ (s, 3H, OMe), 3.50-3.20 (m, 6H, $\left.\mathrm{CH}_{2}\right), 3.01-2.66\left(\mathrm{~m}, 12 \mathrm{H}, \mathrm{CH}_{2}\right)$, 2.37-1.94 (m, 6H, $\left.\mathrm{CH}_{2}\right), 1.41\left(\mathrm{~s}, 9 \mathrm{H},{ }^{\mathrm{t}} \mathrm{Bu}\right), 1.26\left(\mathrm{~s}, 9 \mathrm{H},{ }^{\mathrm{t}} \mathrm{Bu}\right),{ }^{13} \mathrm{C} \mathrm{NMR}\left(100 \mathrm{MHz}, \mathrm{CD}_{3} \mathrm{OD}\right) \delta 161.5,158.2,148.5,131.4$,

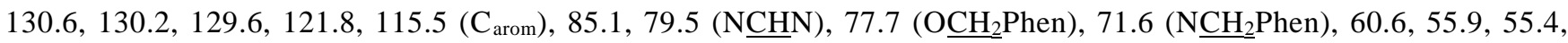
53.7, 53.1, 52.8, 44.8, $43.7\left(\mathrm{CH}_{2}\right.$ and $\left.\mathrm{OMe}\right), 36.7,35.6\left(\mathrm{C}\left({ }^{\mathrm{t}} \mathrm{Bu}\right)\right), 31.9,31.7\left(\mathrm{Me}\left({ }^{\mathrm{t}} \mathrm{Bu}\right)\right), 20.2,19.9\left(\mathrm{CH}_{2} \underline{\mathrm{CH}}_{2} \mathrm{CH}_{2}\right) ; \mathrm{MS}$ (ES-MS): $m / z 562.4\left(\mathrm{M}^{+}\right), 222\left((\text { macrocycle })^{+}\right)$.

Synthesis of 3a-(2-acetoxy-3,5-di-tert-butyl-benzyl)-decahydro-5a,8a,10a-triaza-3a-azonia-pyrene bromide (13)

$12(1.36 \mathrm{~g}, 6.13 \mathrm{mmol})$ and $\mathbf{1 1}(2.09 \mathrm{~g}, 6.13 \mathrm{mmol})$ were dissolved in dry MeCN (15 ml) and stirred at RT for 3 days. After this period, the reaction mixture was concentrated under reduced pressure and the residue taken up in to diethyl ether $(40 \mathrm{ml})$. A white solid formed which was collected by filtration $(2.60 \mathrm{~g}, 75 \%) ;{ }^{1} \mathrm{H} \mathrm{NMR}\left(400 \mathrm{MHz}, \mathrm{CD}_{3} \mathrm{OD}\right) \delta 7.70$ (d, $1 \mathrm{H}, J=2.5 \mathrm{~Hz}, \mathrm{ArH}), 7.48$ (br s, $1 \mathrm{H}, \mathrm{ArH}), 4.85$ (s, 2H, $\left.\mathrm{NCH}_{2} \mathrm{Ph}\right), 4.30$ (br s, 2H, $\left.\mathrm{CH}_{2}\right), 3.65-3.59$ (m, 2H, $\left.\mathrm{CH}_{2}\right), 3.15-$ 2.96 (br m, 8H, $\mathrm{CH}_{2}$ ), 2.69-2.47 (m, 6H, $\left.\mathrm{CH}_{2}\right), 2.38-2.25\left(\mathrm{~m}, 4 \mathrm{H}, \mathrm{CH}_{2}\right), 2.02(\mathrm{~s}, 3 \mathrm{H}, \mathrm{COMe}), 1.23\left(\mathrm{~s}, 9 \mathrm{H},{ }^{\mathrm{t}} \mathrm{Bu}\right), 1.22(\mathrm{~s}$, $\left.9 \mathrm{H},{ }^{\mathrm{t}} \mathrm{Bu}\right) ;{ }^{13} \mathrm{C} \mathrm{NMR}\left(100 \mathrm{MHz}, \mathrm{CD}_{3} \mathrm{OD}\right) \delta 170.5(\mathrm{CO}), 149.7,147.9,143.2,128.4,120.9,117.2\left(\mathrm{C}_{\text {arom }}\right), 70.8(\mathrm{NCHN})$, $59.7\left(\underline{\mathrm{NCH}}_{2} \mathrm{Ph}\right), 54.8,54.6,52.7,52.5,42.9\left(\mathrm{CH}_{2}\right), 35.3,34.9\left(\mathrm{C}\left({ }^{\mathrm{t}} \mathrm{Bu}\right)\right), 30.7,30.2\left(\mathrm{Me}\left({ }^{\mathrm{t}} \mathrm{Bu}\right)\right), 21.4(\mathrm{COOMe}), 19.3,19.2$ $\left(\mathrm{CH}_{2} \underline{\mathrm{CH}}_{2} \mathrm{CH}_{2}\right) ; \mathrm{MS}(\mathrm{ES}-\mathrm{MS}): \mathrm{m} / z 483.3\left(\mathrm{M}^{+}\right)$. 
Synthesis of 5-(3,5-di-tert-butyl-2-methoxybenzyl)-1,5,8,12-tetraazabicyclo[10.2.2]hexadecane $\left(\boldsymbol{L}^{2}\right)$

$14(250 \mathrm{mg}, 0.467 \mathrm{mmol})$ was dissolved in ethanol $(100 \mathrm{ml})$ to which was added $\mathrm{NaBH}_{4}(0.36 \mathrm{~g}, 9.3 \mathrm{mmol})$ portionwise. The solution was then stirred for 7 days at RT before removing the solvent in vacuo. The white solid formed was partitioned between $\mathrm{CH}_{2} \mathrm{Cl}_{2}(60 \mathrm{ml})$ and water $(\mathrm{pH} 14,200 \mathrm{ml})$. The organic layer was collected and dried over $\mathrm{Na}_{2} \mathrm{SO}_{4}$. The solution was then filtered and the filtrate concentrated under reduced pressure to give $\mathbf{L}^{2}$ as a yellow oil (190 $\mathrm{mg}$, 89\%); ${ }^{1} \mathrm{H}$ NMR (400 MHz, $\mathrm{CDCl}_{3}$ ) $\delta 7.16$ (d, $\left.1 \mathrm{H}, J=2.5 \mathrm{~Hz}, \mathrm{ArH}\right), 7.11$ (d, $\left.1 \mathrm{H}, J=2.5 \mathrm{~Hz}, \mathrm{ArH}\right), 3.65$ (s, 3H, OMe), $3.00-$ $2.13\left(\mathrm{~m}, 26 \mathrm{H}, \mathrm{CH}_{2}\right), 1.32$ (s, 9H, $\left.{ }^{\mathrm{t}} \mathrm{Bu}\right), 1.23\left(\mathrm{~s}, 9 \mathrm{H},{ }^{\mathrm{t}} \mathrm{Bu}\right) ;{ }^{13} \mathrm{C} \mathrm{NMR}\left(100 \mathrm{MHz}, \mathrm{CDCl}_{3}\right) \delta$ 156.7, 145.0, 141.8, 126.6, 123.1 $\left(\mathrm{C}_{\text {arom }}\right), 62.4(\mathrm{OMe}), 57.1\left(\mathrm{NCH}_{2} \mathrm{Ph}\right), 57.0,54.5,54.3,53.7,53.4,51.7,51.1,49.8,48.6,48.1\left(\mathrm{CH}_{2}\right), 35.3,34.4\left(\mathrm{C}\left({ }^{\mathrm{t}} \mathrm{Bu}\right)\right)$, 31.6, $31.3\left(\mathrm{Me}\left({ }^{\mathrm{t}} \mathrm{Bu}\right)\right), 24.0,19.6\left(\mathrm{NCH}_{2} \underline{\mathrm{CH}}_{2} \mathrm{CH}_{2} \mathrm{~N}\right)$; MS (ES-MS): $\mathrm{m} / z 460.5\left(\mathrm{M}^{+}\right)$.

Synthesis of 5-(2-(benzyloxy)-3,5-di-tert-butylbenzyl)-1,5,8,12-tetraazabicyclo[10.2.2]hexadecane ( $\left.\boldsymbol{L}^{3}\right)$

$15(1.09 \mathrm{~g}, 0.00178 \mathrm{~mol})$ was dissolved in methanol $(40 \mathrm{ml})$ to which $\mathrm{NaBH}_{4}(1.36 \mathrm{~g}, 0.03568 \mathrm{~mol})$ was added portionwise over $5 \mathrm{~min}$. The mixture was then stirred at RT for 2 days before heating to reflux for $2 \mathrm{hr}$. The solvent was removed under reduced pressure and $\mathrm{KOH}_{(\mathrm{aq})}(\mathrm{pH} 14,200 \mathrm{ml})$ added. $\mathrm{CH}_{2} \mathrm{Cl}_{2}(100 \mathrm{ml})$ was used to extract the organic product which was then dried over $\mathrm{MgSO}_{4}$ and evaporated in vacuo to afford $\mathbf{L}^{3}$ as a yellow oil $(0.76 \mathrm{~g}, 80 \%) ;{ }^{1} \mathrm{H} \mathrm{NMR}$ (400 MHz, $\left.\mathrm{CDCl}_{3}\right) \delta$ 7.44-7.20 (m, 7H, ArH), 5.19 (s, 2H, $\mathrm{NCH}_{2} \mathrm{Bn}$ ), $4.82\left(\mathrm{~s}, 2 \mathrm{H}, \mathrm{OCH}_{2} \mathrm{Bn}\right), 3.70-3.68\left(\mathrm{~m}, 2 \mathrm{H}, \mathrm{CH}_{2}\right)$, 3.28-3.24 (m, 7H, $\left.\mathrm{CH}_{2}\right), 3.12-2.84\left(\mathrm{~m}, 5 \mathrm{H}, \mathrm{CH}_{2}\right), 2.56-2.39\left(\mathrm{~m}, 11 \mathrm{H}, \mathrm{CH}_{2}\right), 1.36\left(\mathrm{~s}, 9 \mathrm{H},{ }^{\mathrm{t}} \mathrm{Bu}\right), 1.23\left(\mathrm{~s}, 9 \mathrm{H},{ }^{\mathrm{t}} \mathrm{Bu}\right) ;{ }^{13} \mathrm{C} \mathrm{NMR}$ $\left(100 \mathrm{MHz}, \mathrm{CDCl}_{3}\right) 155.0,145.4,142.0,137.9,129.9,128.5,127.6,126.7,126.6,123.3\left(\mathrm{C}_{\text {arom }}\right), 77.0\left(\mathrm{OCH}_{2} \mathrm{Ph}\right), 56.9$ $\left(\mathrm{NCH}_{2} \mathrm{Ph}\right), 54.5,54.0,53.6,51.0,50.7,49.7,48.2,47.8\left(\mathrm{CH}_{2}\right), 35.4,34.5\left(\mathrm{C}\left({ }^{\mathrm{t}} \mathrm{Bu}\right)\right), 31.6,31.3\left(\mathrm{Me}\left({ }^{(} \mathrm{Bu}\right)\right), 25.6,23.8$ $\left(\mathrm{NCH}_{2} \mathrm{CH}_{2} \mathrm{CH}_{2} \mathrm{~N}\right) ; \mathrm{MS}$ (ES-MS): $\mathrm{m} / 2536.4\left(\mathrm{MH}^{+}\right)$.

Synthesis of 5-[3,5-di-tert-butyl-2-(4-methoxy-benzyloxy)-benzyl]-1,5,8,12-tetraaza-bicyclo[10.2.2]hexadecane ( $\left.\boldsymbol{L}^{4}\right)$

To 16 (1 g, $1.56 \mathrm{mmol})$ dissolved in EtOH (50 ml) was added $\mathrm{NaBH}_{4}(1.18 \mathrm{~g}, 31.20 \mathrm{mmol})$ portionwise. A colour change from yellow to colourless occurred during this addition. The mixture was then left for 2 weeks stirring at RT, before concentrating under reduced pressure. The residual solid was then partitioned between $\mathrm{CH}_{2} \mathrm{Cl}_{2}(50 \mathrm{ml})$ and $\mathrm{KOH}_{(\mathrm{aq})}(\mathrm{pH}$ $14,200 \mathrm{ml})$. The organic layer was then collected and dried over $\mathrm{MgSO}_{4}$ before concentrating under reduced pressure. The product was obtained as a yellow oil $(0.85 \mathrm{~g}, 97 \%) ;{ }^{1} \mathrm{H} \mathrm{NMR}\left(400 \mathrm{MHz}, \mathrm{CDCl}_{3}\right) \delta 7.37(\mathrm{~d}, 2 \mathrm{H}, J=8.4 \mathrm{~Hz}, \mathrm{ArH})$, $7.32(\mathrm{~s}, 1 \mathrm{H}, \mathrm{ArH}), 7.23$ (s, 1H, ArH), $6.86(\mathrm{~d}, 2 \mathrm{H}, J=8.4 \mathrm{~Hz}, \mathrm{ArH}), 4.73$ (s, 2H, $\left.\mathrm{OCH}_{2} \mathrm{Phen}\right), 3.70$ (s, 3H, OMe), 3.65 (s, $2 \mathrm{H}, \mathrm{NCH}_{2} \mathrm{Phen}$ ), 3.05-2.99 (m, 1H, $\left.\mathrm{CH}_{2}\right), 2.78$ (br s, 6H, CH 2$), 2.56-2.35\left(\mathrm{~m}, 12 \mathrm{H}, \mathrm{CH}_{2}\right), 2.21-1.94\left(\mathrm{~m}, 6 \mathrm{H}, \mathrm{CH}_{2}\right), 1.37$ (s, 9H, $\left.{ }^{\mathrm{t}} \mathrm{Bu}\right), 1.29\left(\mathrm{~s}, 9 \mathrm{H},{ }^{\mathrm{t}} \mathrm{Bu}\right){ }^{13} \mathrm{C} \mathrm{NMR}\left(100 \mathrm{MHz}, \mathrm{CDCl}_{3}\right) \delta 163.6,159.2,149.5,145.9,135.6,134.3,132.8,130.6$, 127.1, $117.8\left(\mathrm{C}_{\text {arom }}\right), 81.5\left(\underline{\mathrm{OCH}}_{2} \mathrm{Phen}\right), 61.4,59.4,58.2,58.1,57.8,57.5,55.6,53.6,52.4,49.3\left(\mathrm{CH}_{2}\right.$ and $\left.\mathrm{OMe}\right), 39.6$, $38.7\left(\mathrm{C}\left({ }^{\mathrm{H}} \mathrm{Bu}\right)\right), 35.8,35.5\left(\mathrm{Me}\left({ }^{\mathrm{B}} \mathrm{Bu}\right)\right), 24.3,23.01\left(\mathrm{CH}_{2} \mathrm{CH}_{2} \mathrm{CH}_{2}\right)$; MS (ES-MS): $m / z 566.3\left(\mathrm{MH}^{+}\right)$.

Synthesis of acetic acid 2,4-di-tert-butyl-6-(1,5,8,12-tetraaza-bicyclo[10.2.2]hexadec-5-ylmethyl)phenyl ester (17)

$13(2.6 \mathrm{~g}, 4.62 \mathrm{mmol})$ was dissolved in ethanol $(150 \mathrm{ml})$ to which was added $\mathrm{NaBH}_{4}(3.51 \mathrm{~g}, 92.4 \mathrm{mmol})$ portion wise. The reaction mixture was stirred at RT overnight at which time hydrogen evolution had ceased. The reaction mixture was then concentrated under reduced pressure and the residue partitioned between $\mathrm{CH}_{2} \mathrm{Cl}_{2}(100 \mathrm{ml})$ and $\mathrm{KOH}_{(\mathrm{aq})}(\mathrm{pH} 14$, $200 \mathrm{ml}$ ). The organic layer was then collected and dried over $\mathrm{MgSO}_{4}$. The filtrate was then concentrated under reduced pressure before dissolving in the minimal amount of acetone $(\sim 10 \mathrm{ml})$ which was triturated with pentane $(150 \mathrm{ml})$ to give a cream solid which was collected by filtration $(2.20 \mathrm{~g}, 98 \%) ;{ }^{1} \mathrm{H} \mathrm{NMR}\left(400 \mathrm{MHz}, \mathrm{CDCl}_{3}\right) \delta 7.37(\mathrm{~d}, 2 \mathrm{H}, \mathrm{J}=2.5 \mathrm{~Hz}$, $\mathrm{ArH}), 7.24$ (d, 2H, J=2.5 Hz, ArH), 5.23 (s, 2H, $\mathrm{CH}_{2}$ ), 3.33-2.37 (m, 28H, $\mathrm{CH}_{2}$ and $\mathrm{NH}$ ), 2.25 (s, 3H, COOMe), 1.27 (s, $\left.9 \mathrm{H},{ }^{\mathrm{t}} \mathrm{Bu}\right), 1.25\left(\mathrm{~s}, 9 \mathrm{H},{ }^{\mathrm{t}} \mathrm{Bu}\right) ;{ }^{13} \mathrm{C} \mathrm{NMR}\left(100 \mathrm{MHz}, \mathrm{CDCl}_{3}\right) \delta 166.1$ ( $\left.\underline{\mathrm{CO}}\right), 144.1,142.3,136.8,127.9,122.0,119.3\left(\mathrm{C}_{\text {arom }}\right)$, 53.8, 53.4, 51.4, 49.9, 48.3, 47.9, 45.0, 46.0, 45.5, 44.8, $43.5\left(\mathrm{CH}_{2}\right), 31.2,31.1\left(\mathrm{C}\left({ }^{\mathrm{t}} \mathrm{Bu}\right)\right), 27.7,26.8\left(\mathrm{Me}\left({ }^{\mathrm{t}} \mathrm{Bu}\right)\right), 23.1$, 
22.8, $\left(\mathrm{CH}_{2} \underline{\mathrm{CH}_{2}} \mathrm{CH}_{2}\right), 20.8$ (COOMe); HRMS (ES-MS) expected for $\mathrm{C}_{29} \mathrm{H}_{50} \mathrm{~N}_{4} \mathrm{O}_{2} \mathrm{H}: 487.4007$, found 487.3996.

Synthesis of 2,4-di-tert-butyl-6-(1,5,8,12-tetraaza-bicyclo[10.2.2] hexadec-5-ylmethyl)-phenol hydrochloride salt ( $\left.\boldsymbol{L}^{1}\right)$

To 17 (1.5 g, $3.09 \mathrm{mmol})$ dissolved in $\mathrm{MeOH}(10 \mathrm{ml})$ was added $6 \mathrm{M} \mathrm{HCl}_{(\mathrm{aq})}(10 \mathrm{ml})$. This induced a colour change from yellow to colourless. The solution was then heated to reflux for $24 \mathrm{hr}$ before cooling to room temperature. The solution was concentrated under reduced pressure, and the addition of acetone $(30 \mathrm{ml})$ caused a yellow precipitate to form. This was collected by filtration and dried in vacuo $(1.44 \mathrm{~g}, 79 \%) ;{ }^{1} \mathrm{H}$ NMR $\left(400 \mathrm{MHz}, \mathrm{D}_{2} \mathrm{O}\right) \delta 7.24(\mathrm{~s}, 1 \mathrm{H}, \mathrm{ArH}), 7.16(\mathrm{~s}, 1 \mathrm{H}$, $\mathrm{ArH}), 3.40-3.14\left(\mathrm{~m}, 15 \mathrm{H}, \mathrm{CH}_{2}\right.$ and $\left.\mathrm{NH}\right), 2.02-1.80\left(\mathrm{~m}, 10 \mathrm{H}, \mathrm{CH}_{2}\right), 1.11\left(\mathrm{~s}, 9 \mathrm{H},{ }^{\mathrm{t}} \mathrm{Bu}\right), 1.01\left(\mathrm{~s}, 9 \mathrm{H},{ }^{\mathrm{t}} \mathrm{Bu}\right) ;{ }^{13} \mathrm{C} \mathrm{NMR}(100$ $\left.\mathrm{MHz}, \mathrm{D}_{2} \mathrm{O}\right) \delta 151.7,144.4,140.1,127.7,125.6,120.5$ ( $\left.\mathrm{C}_{\text {arom }}\right), 74.3\left(\mathrm{NCH}_{2} \mathrm{Phen}\right), 49.2,48.8,48.2,48.0,47.8,46.8,46.0$ $\left(\mathrm{CH}_{2}\right)$, 34.6, $33.9\left(\mathrm{C}\left({ }^{\mathrm{t}} \mathrm{Bu}\right)\right), 30.7,29.5\left(\mathrm{Me}\left({ }^{\mathrm{t}} \mathrm{Bu}\right)\right), 21.5,20.7\left(\mathrm{CH}_{2} \underline{\mathrm{CH}}_{2} \mathrm{CH}_{2}\right)$; HRMS (ES-MS) expected for $\mathrm{C}_{27} \mathrm{H}_{48} \mathrm{~N}_{4} \mathrm{OH}$ : 445.3901 , found 445.3891 .

Synthesis of copper(II) 5-(3,5-di-tert-butyl-2-methoxybenzyl)-1,5,8,12-tetraazabicyclo[10.2.2] hexadecane perchlorate $\left(\left[\mathrm{CuL}^{2}\right]\left(\mathrm{ClO}_{4}\right)_{2}\right)$

To a solution of $\mathbf{L}^{2}(100 \mathrm{mg}, 0.218 \mathrm{mmol})$ in $\mathrm{MeOH}(10 \mathrm{ml})$ was added copper(II) perchlorate hexahydrate $(0.08 \mathrm{~g}, 0.218$ $\mathrm{mmol}$ ) in $\mathrm{MeOH}(3 \mathrm{ml})$. The blue solution was then heated to reflux for $2 \mathrm{hr}$. After this time the solution was allowed to cool, filtered and concentrated under reduced pressure to give a blue-purple crystalline solid (154 mg, 98\%); HRMS (ES-MS) expected for $\mathrm{C}_{28} \mathrm{~N}_{4} \mathrm{H}_{50} \mathrm{O}_{5} \mathrm{Cl}_{1} \mathrm{Cu}_{1}: 620.2760$, found 620.2767; $\varepsilon(\mathrm{MeOH}): 547 \mathrm{~nm}\left(331 \mathrm{~mol}^{-1} \mathrm{dm}^{3} \mathrm{~cm}^{-1}\right)$.

Synthesis of zinc(II) 5-(3,5-di-tert-butyl-2-methoxybenzyl)-1,5,8,12-tetraazabicyclo[10.2.2]hexadecane perchlorate $\left(\left[\mathrm{ZnL}^{2}\right]\left(\mathrm{ClO}_{4}\right)_{2}\right)$

To a solution of $\mathbf{L}^{2}(100 \mathrm{mg}, 0.218 \mathrm{mmol})$ in $\mathrm{MeOH}(10 \mathrm{ml})$ was added zinc(II) perchlorate hexahydrate $(0.08 \mathrm{~g}, 0.218$ mmol) in $\mathrm{MeOH}(3 \mathrm{ml})$. The yellow solution was then heated to reflux for $2 \mathrm{hr}$. After this time the solution was allowed to cool, filtered and concentrated under reduced pressure to give a white crystalline solid (150 mg, 95\%); ${ }^{1} \mathrm{H}$ NMR $(400$ $\left.\mathrm{MHz}, \mathrm{CD}_{3} \mathrm{CN}\right) \delta 7.14(\mathrm{~d}, 1 \mathrm{H}, J=2.5 \mathrm{~Hz}, \mathrm{ArH}), 7.10(\mathrm{~d}, 1 \mathrm{H}, J=2.5 \mathrm{~Hz}, \mathrm{ArH}), 3.64(\mathrm{~s}, 3 \mathrm{H}, \mathrm{OMe}), 2.99-2.11\left(\mathrm{~m}, 26 \mathrm{H}, \mathrm{CH}_{2}\right)$, $1.33\left(\mathrm{~s}, 9 \mathrm{H},{ }^{\mathrm{t}} \mathrm{Bu}\right), 1.25\left(\mathrm{~s}, 9 \mathrm{H},{ }^{\mathrm{t}} \mathrm{Bu}\right) ;{ }^{13} \mathrm{C} \mathrm{NMR}\left(400 \mathrm{MHz}, \mathrm{CD}_{3} \mathrm{CN}\right) \delta 157.5,145.1,141.5,125.7,124.3\left(\mathrm{C}_{\text {arom }}\right), 62.9$ $(\mathrm{OMe}), 57.8\left(\mathrm{NCH}_{2} \mathrm{Ph}\right), 56.5,54.4,54.3,53.7,53.3,51.6,51.2,49.8,48.7,48.1\left(\mathrm{CH}_{2}\right), 35.5,34.7\left(\mathrm{C}\left({ }^{\mathrm{t}} \mathrm{Bu}\right)\right), 31.6,31.6$ (Me( $\left.\left.{ }^{\mathrm{t}} \mathrm{Bu}\right)\right), 24.1,19.6\left(\mathrm{NCH}_{2} \underline{\mathrm{CH}}_{2} \mathrm{CH}_{2} \mathrm{~N}\right)$; HRMS (ES-MS) expected for $\mathrm{C}_{28} \mathrm{H}_{50} \mathrm{O}_{5} \mathrm{~N}_{4} \mathrm{Cl}_{1} \mathrm{Zn}_{1}$ : 621.2756, found 621.2763.

Synthesis of nickel(II) 5-(3,5-di-tert-butyl-2-methoxybenzyl)-1,5,8,12-tetraazabicyclo[10.2.2]hexadecane perchlorate $\left(\left[\mathrm{NiL}^{2}\right]\left(\mathrm{ClO}_{4}\right)_{2}\right)$

To a solution of $\mathbf{L}^{2}(100 \mathrm{mg}, 0.218 \mathrm{mmol})$ in $\mathrm{MeOH}(10 \mathrm{ml})$ was added nickel(II) perchlorate hexahydrate (0.08 $\mathrm{g}, 0.218$ $\mathrm{mmol})$ in $\mathrm{MeOH}(3 \mathrm{ml})$. The orange solution was then heated to reflux for $2 \mathrm{hr}$. After this time the solution was allowed to cool, filtered and concentrated under reduced pressure to give a orange crystalline solid (152 mg, 98\%); HRMS (ES MS) expected for $\mathrm{C}_{28} \mathrm{H}_{50} \mathrm{~N}_{4} \mathrm{ONi}$ : $258.1664\left(\mathrm{M}^{2+}\right)$ found 258.1665; HRMS (ES-MS) expected for $\mathrm{C}_{28} \mathrm{H}_{50} \mathrm{~N}_{4} \mathrm{ONi}$ : 615.2818 $\left(\mathrm{M}^{+}\right)$found 615.2808; $\varepsilon(\mathrm{MeOH}): 477 \mathrm{~nm}\left(197 \mathrm{~mol}^{-1} \mathrm{dm}^{3} \mathrm{~cm}^{-1}\right)$.

Synthesis of copper(II) 5-(2-(benzyloxy)-3,5-di-tert-butylbenzyl)-1,5,8,12-tetraazabicyclo[10.2.2]hexadecane perchlorate $\left(\left[\mathrm{CuL}^{3}\right]\left(\mathrm{ClO}_{4}\right)_{2}\right)$

To $\mathbf{L}^{3}$ (100 mg, $\left.0.187 \mathrm{mmol}\right)$ dissolved in $\mathrm{MeOH}(10 \mathrm{ml})$ was added copper(II) perchlorate hexahydrate $(0.07 \mathrm{~g}, 0.187$ $\mathrm{mmol})$ in $\mathrm{MeOH}(3 \mathrm{ml})$. The blue solution was then heated to reflux for $2 \mathrm{hr}$. After this time the solution was allowed to cool, filtered and concentrated under reduced pressure to give a purple crystalline solid (130 mg, 87\%); HRMS (ES-MS) expected for $\mathrm{C}_{34} \mathrm{H}_{54} \mathrm{O}_{5} \mathrm{~N}_{4} \mathrm{Cl}_{1} \mathrm{Cu}_{1}: 696.3073$, found 696.3075; $\varepsilon(\mathrm{MeOH}): 550 \mathrm{~nm}\left(286 \mathrm{~mol}^{-1} \mathrm{dm}^{3} \mathrm{~cm}^{-1}\right)$. 
Synthesis of zinc(II) 5-(2-(benzyloxy)-3,5-di-tert-butylbenzyl)-1,5,8,12-tetraazabicyclo[10.2.2]hexadecane perchlorate $\left(\left[\mathrm{ZnL} \mathbf{L}^{3}\right]\left(\mathrm{ClO}_{4}\right)_{2}\right)$

To $\mathbf{L}^{3}$ (100 mg, $\left.0.187 \mathrm{mmol}\right)$ dissolved in $\mathrm{MeOH}(10 \mathrm{ml})$ was added zinc(II) perchlorate hexahydrate $(0.0822 \mathrm{~g}, 0.187$ $\mathrm{mmol})$ in $\mathrm{MeOH}(3 \mathrm{ml})$. The yellow solution was then heated to reflux for $2 \mathrm{hr}$. After this time the solution was allowed to cool, filtered and concentrated under reduced pressure to give a white crystalline solid $(0.0702 \mathrm{mg}, 47 \%)$; ${ }^{1} \mathrm{H} \mathrm{NMR}$ $\left(400 \mathrm{MHz}, \mathrm{CD}_{3} \mathrm{CN}\right) \delta$ 7.46-7.22 (m, 7H, ArH), 5.21 (s, 2H, $\left.\mathrm{NCH}_{2} \mathrm{Bn}\right), 4.79$ (s, 2H, OCH$\left.{ }_{2} \mathrm{Bn}\right), 3.72-3.66\left(\mathrm{~m}, 2 \mathrm{H}, \mathrm{CH}_{2}\right)$, 3.29-3.22 (m, 7H, $\left.\mathrm{CH}_{2}\right), 3.13-2.82\left(\mathrm{~m}, 5 \mathrm{H}, \mathrm{CH}_{2}\right), 2.57-2.33\left(\mathrm{~m}, 11 \mathrm{H}, \mathrm{CH}_{2}\right), 1.34\left(\mathrm{~s}, 9 \mathrm{H},{ }^{\mathrm{t}} \mathrm{Bu}\right), 1.26\left(\mathrm{~s}, 9 \mathrm{H},{ }^{\mathrm{t}} \mathrm{Bu}\right) ;{ }^{13} \mathrm{C} \mathrm{NMR}$ $\left(100 \mathrm{MHz}, \mathrm{CD}_{3} \mathrm{CN}\right) 156.1,144.4,142.0,137.6,129.6,128.8,127.5,126.6,126.4,123.3\left(\mathrm{C}_{\text {arom }}\right), 77.1(\mathrm{OCH} \underline{2} \underline{\mathrm{Ph}}), 56.4$ $\left(\underline{\mathrm{NCH}}_{2} \mathrm{Ph}\right), 54.5,53.8,53.6,51.1,50.7,49.6,48.2,47.9\left(\mathrm{CH}_{2}\right), 35.3,34.7\left(\mathrm{C}\left({ }^{\mathrm{t}} \mathrm{Bu}\right)\right), 31.6,31.3\left(\mathrm{Me}\left({ }^{\mathrm{t}} \mathrm{Bu}\right)\right), 25.6,23.8$ $\left(\mathrm{NCH}_{2} \underline{\mathrm{CH}_{2}} \mathrm{CH}_{2} \mathrm{~N}\right)$; HRMS (ES-MS) expected for $\mathrm{C}_{34} \mathrm{H}_{54} \mathrm{~N}_{4} \mathrm{O}_{5} \mathrm{ZnCl}$ : 697.3069, found 697.3060.

Synthesis of nickel(II) 5-(2-(benzyloxy)-3,5-di-tert-butylbenzyl)-1,5,8,12-tetraazabicyclo[10.2.2] hexadecane perchlorate $\left(\left[\mathrm{NiL}^{3}\right]\left(\mathrm{ClO}_{4}\right)_{2}\right)$

To $\mathbf{L}^{3}$ (150 mg, $\left.0.281 \mathrm{mmol}\right)$ dissolved in $\mathrm{MeOH}$ (10 ml) was added nickel(II) perchlorate hexahydrate (102 $\mathrm{mg}, 0.281$ $\mathrm{mmol})$ in $\mathrm{MeOH}(3 \mathrm{ml})$. The yellow/brown solution was then heated to reflux for $2 \mathrm{hr}$. After this time the solution was allowed to cool, filtered and concentrated under reduced pressure to give an orange crystalline solid $(0.0925 \mathrm{~g}, 42 \%)$;

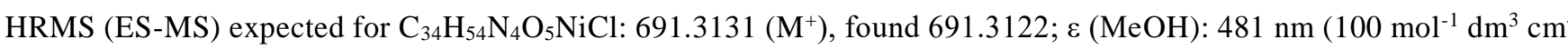
$1)$.

Synthesis of copper(II) 5-[3,5-di-tert-butyl-2-(4-methoxy-benzyloxy)-benzyl]-1,5,8,12-tetraazabicyclo[10.2.2] hexadecane perchlorate $\left(\left[\mathrm{CuL}^{4}\right]\left(\mathrm{ClO}_{4}\right)_{2}\right.$

To $\mathbf{L}^{4}(0.1 \mathrm{~g}, 0.1773 \mathrm{mmol})$ dissolved in $\mathrm{MeOH}(8 \mathrm{ml})$ was added copper(II) perchlorate hexahydrate $(0.657 \mathrm{~g}, 0.1773$ $\mathrm{mmol})$ in $\mathrm{MeOH}(2 \mathrm{ml})$. The blue solution was heated to reflux for $2 \mathrm{hr}$ before it was allowed to cool to RT. The solution was then concentrated under reduced pressure before redissolving in the minimal amount of MeOH. This solution was then eluted down a Sephadex LH-20 column with $\mathrm{MeOH}$. The blue fraction was isolated and concentrated under reduced pressure. The oily residue was triturated with ether $(3 \times 5 \mathrm{ml})$ to afford the title compound as a blue solid (0.0430 $\mathrm{g}$, 29\%); HRMS (ES-MS) expected for $\mathrm{C}_{35} \mathrm{H}_{56} \mathrm{O}_{2} \mathrm{~N}_{4} \mathrm{CuClO}_{4}: 726.3179$, found 726.3183; $\varepsilon(\mathrm{MeOH}): 529 \mathrm{~nm}^{\left(154 \mathrm{~mol}^{-1} \mathrm{dm}\right.}$ $\left.\mathrm{cm}^{-1}\right)$.

Synthesis of nickel(II) 5-[3,5-di-tert-butyl-2-(4-methoxy-benzyloxy)-benzyl]-1,5,8,12-tetraazabicyclo[10.2.2] hexadecane perchlorate $\left(\left[\mathrm{NiL}^{4}\right]\left(\mathrm{ClO}_{4}\right)_{2}\right)$

To $\mathbf{L}^{4}(0.1 \mathrm{~g}, 0.1773 \mathrm{mmol})$ dissolved in $\mathrm{MeOH}(8 \mathrm{ml})$ was added nickel(II) hexahydrate $(0.0648 \mathrm{~g}, 0.1773 \mathrm{mmol}) \mathrm{in}$ $\mathrm{MeOH}(2 \mathrm{ml})$. The orange solution was heated to reflux for $2 \mathrm{hr}$ before it was allowed to cool to RT. The solution was then concentrated under reduced pressure before redissolving in the minimal amount of $\mathrm{MeOH}$. This solution was then eluted on a Sephadex LH-20 column with $\mathrm{MeOH}$. The orange fraction was isolated and concentrated under reduced pressure. The oily residue was triturated with ether $(3 \times 5 \mathrm{ml})$ to afford the title compound as an orange solid $(0.0640 \mathrm{~g}$, 44\%); HRMS (ES-MS) expected for $\mathrm{C}_{35} \mathrm{H}_{56} \mathrm{O}_{2} \mathrm{~N}_{4} \mathrm{NiClO}_{4}: 721.3236$, found 721.3237; $\varepsilon(\mathrm{MeOH}): 478 \mathrm{~nm}^{(96} \mathrm{mol}^{-1} \mathrm{dm}^{3}$ $\left.\mathrm{cm}^{-1}\right)$.

Synthesis of zinc(II) 5-[3,5-di-tert-butyl-2-(4-methoxy-benzyloxy)-benzyl]-1,5,8,12-tetraaza-bicyclo[10.2.2]hexadecane perchlorate $\left(\left[\mathrm{ZnL}^{4}\right]\left(\mathrm{ClO}_{4}\right)_{2}\right)$

To $\mathbf{L}^{4}(0.1 \mathrm{~g}, 0.1773 \mathrm{mmol})$ dissolved in $\mathrm{MeOH}(8 \mathrm{ml})$ was added zinc(II) hexahydrate $(0.066 \mathrm{~g}, 0.1773 \mathrm{mmol}) \mathrm{in} \mathrm{MeOH}$ $(2 \mathrm{ml})$. The yellow solution was heated to reflux for $2 \mathrm{hr}$ before it was allowed to cool to RT. The solution was then 
concentrated under reduced pressure before redissolving in the minimal amount of $\mathrm{MeOH}$. This solution was then eluted on a Sephadex LH-20 column with $\mathrm{MeOH}$. The light yellow fraction was isolated and concentrated under reduced pressure. The oily residue was triturated with ether $(3 \times 5 \mathrm{ml})$ to afford the title compound as a white solid $(0.0720 \mathrm{~g}$, 49\%); ${ }^{1} \mathrm{H}$ NMR (400 MHz, CD 3 CN) 8.35 (d, 2H, J=8.7 Hz, ArH), 7.29 (s, 1H, ArH), 7.21 (br s, 1H, ArH), 6.86 (d, 2H, $J=8.7 \mathrm{~Hz}, \mathrm{ArH}$ ), 4.77 (s, 2H, $\mathrm{OCH}_{2} \mathrm{Phen}$ ), 3.69 (s, 3H, OMe), 3.63 (s, $\left.2 \mathrm{H}, \mathrm{NCH}_{2} \mathrm{Phen}\right), 3.11-3.01\left(\mathrm{~m}, 1 \mathrm{H}, \mathrm{CH}_{2}\right), 2.78$ (br $\left.\mathrm{s}, 6 \mathrm{H}, \mathrm{CH}_{2}\right), 2.56-1.92\left(\mathrm{~m}, 18 \mathrm{H}, \mathrm{CH}_{2}\right), 1.34\left(\mathrm{~s}, 9 \mathrm{H},{ }^{\mathrm{t}} \mathrm{Bu}\right), 1.26\left(\mathrm{~s}, 9 \mathrm{H},{ }^{\mathrm{t}} \mathrm{Bu}\right) ;{ }^{13} \mathrm{C}$ NMR $\left(100 \mathrm{MHz}, \mathrm{CDCl}_{3}\right) \delta 164.0,158.9$, 149.2, 146.1, 135.8, 134.3, 132.7, 130.2, 127.4, $117.6\left(\mathrm{C}_{\text {arom }}\right), 81.5\left(\mathrm{OCH}_{2} \mathrm{Phen}\right), 60.5,59.3,58.2,58.0,57.8,56.3,55.8$, 54.6, 52.8, $49.4\left(\mathrm{CH}_{2}\right.$ and $\left.\mathrm{OMe}\right), 39.5,38.6\left(\mathrm{C}\left({ }^{\mathrm{t}} \mathrm{Bu}\right)\right), 35.7,35.5\left(\mathrm{Me}\left({ }^{\mathrm{t}} \mathrm{Bu}\right)\right), 24.7,23.2\left(\mathrm{CH}_{2} \mathrm{CH}_{2} \mathrm{CH}_{2}\right)$; HRMS (ES) expected for $\mathrm{C}_{34} \mathrm{H}_{54} \mathrm{O}_{2} \mathrm{~N}_{4} \mathrm{ZnO}_{4} \mathrm{Cl}$ : 713.3804, found 713.3807.

Synthesis of copper(II) 2,4-di-tert-butyl-6-(1,5,8,12-tetraaza-bicyclo[10.2.2] hexadec-5-ylmethyl)-phenolate perchlorate ([ $\left.\left.\mathrm{CuL}^{1}\right] \mathrm{ClO}_{4}\right)$

To $\mathbf{L}^{1}(180 \mathrm{mg}, 0.305 \mathrm{mmol})$ in $\mathrm{MeOH}(8 \mathrm{ml})$ was added $\mathrm{NEt}_{3}(150 \mathrm{mg}, 1.5 \mathrm{mmol})$. The reaction mixture was stirred for 5 min, by which time the colour of the solution had changed from yellow to straw colour. To this solution was then added copper(II) perchlorate hexahydrate $(113 \mathrm{mg}, 0.305 \mathrm{mmol})$ in $\mathrm{MeOH}(3 \mathrm{ml})$. The royal blue solution was then heated to reflux for $2 \mathrm{hr}$ before it was allowed to cool. The reaction mixture was concentrated under reduced pressure to a minimal volume before eluting down a Sephadex LH-20 column using MeOH. The blue fraction was collected and concentrated under reduced pressure. The oil obtained was triturated with ether $(3 \times 5 \mathrm{ml})$ to give a hygroscopic blue solid $(0.0737 \mathrm{~g}$, 40\%); HRMS (ES-MS) expected for (cation-2H) ${ }^{+} \mathrm{C}_{27} \mathrm{H}_{45} \mathrm{~N}_{4} \mathrm{O}_{1} \mathrm{Cu}_{1}$ : 504.2884 found 504.2875; $\varepsilon$ (MeOH): $641 \mathrm{~nm}$ (27 $\left.\mathrm{mol}^{-1} \mathrm{dm}^{3} \mathrm{~cm}^{-1}\right)$.

Synthesis of nickel(II) 2,4-di-tert-butyl-6-(1,5,8,12-tetraaza-bicyclo[10.2.2]hexadec-5-ylmethyl)-phenolate perchlorate $\left(\left[\mathrm{NiL}^{1}\right] \mathrm{ClO}_{4}\right)$

To $\mathbf{L}^{1}$ (180 mg, $\left.0.305 \mathrm{mmol}\right)$ in $\mathrm{MeOH}(8 \mathrm{ml})$ was added $\mathrm{NEt}_{3}(150 \mathrm{mg}, 1.5 \mathrm{mmol})$. The reaction mixture was stirred for 5 min, by which time the colour of the solution had changed from yellow to straw colour. To this solution was then added nickel(II) perchlorate hexahydrate $(112 \mathrm{mg}, 0.305 \mathrm{mmol})$ in $\mathrm{MeOH}(3 \mathrm{ml})$. The orange solution was then heated to reflux for $2 \mathrm{hr}$ before it was allowed to cool. The reaction mixture was concentrated under reduced pressure to a minimal volume before eluting down a Sephadex LH-20 column using MeOH. The orange fraction was collected and concentrated under reduced pressure. The oil obtained was triturated with ether $(3 \times 5 \mathrm{ml})$ to give a hygroscopic orange solid $(0.0541$ g, 30\%); HRMS (ES) expected for $\mathrm{C}_{27} \mathrm{H}_{47} \mathrm{~N}_{4} \mathrm{ONi}$ : 501.3098, found 501.3093; $\varepsilon(\mathrm{MeOH}): 441 \mathrm{~nm}\left(12 \mathrm{~mol}^{-1} \mathrm{dm}^{3} \mathrm{~cm}^{-1}\right)$.

Synthesis of zinc(II) 2,4-di-tert-butyl-6-(1,5,8,12-tetraaza-bicyclo[10.2.2] hexadec-5-ylmethyl)-phenolate perchlorate $\left(\left[\mathrm{ZnL}^{1}\right] \mathrm{ClO}_{4}\right)$

To $\mathbf{L}^{1}$ (180 mg, $\left.0.305 \mathrm{mmol}\right)$ in $\mathrm{MeOH}(8 \mathrm{ml})$ was added $\mathrm{NEt}_{3}(150 \mathrm{mg}, 1.5 \mathrm{mmol})$. The reaction mixture was stirred for 5 min, by which time the colour of the solution had changed from yellow to straw colour. To this solution was then added zinc(II) perchlorate hexahydrate $(113 \mathrm{mg}, 0.305 \mathrm{mmol})$ in $\mathrm{MeOH}(3 \mathrm{ml})$. The straw coloured solution was then heated to reflux for $2 \mathrm{hr}$ before it was allowed to cool. The reaction mixture was concentrated under reduced pressure to a minimal volume before eluting down a Sephadex LH-20 column using $\mathrm{MeOH}$ as the eluent. The yellow fraction was collected and concentrated under reduced pressure. The oil obtained was triturated with ether $(3 \times 5 \mathrm{ml})$ to give a hygroscopic white solid (0.0530 g, 29\%); ${ }^{1} \mathrm{H}$ NMR (400 MHz, $\left.\mathrm{CD}_{3} \mathrm{CN}\right) \delta 7.63$ (s, 1H, ArH), $7.53(\mathrm{~s}, 1 \mathrm{H}, \mathrm{ArH}), 4.10-$ $4.09\left(\mathrm{~m}, 2 \mathrm{H}, \mathrm{CH}_{2} \mathrm{Ph}\right), 3.26-2.09\left(\mathrm{~m}, 25 \mathrm{H}, \mathrm{NH}\right.$ and $\left.\mathrm{CH}_{2}\right), 1.31\left(\mathrm{~s}, 9 \mathrm{H},{ }^{\mathrm{t}} \mathrm{Bu}\right), 1.22\left(\mathrm{~s}, 9 \mathrm{H},{ }^{\mathrm{t}} \mathrm{Bu}\right) ;{ }^{13} \mathrm{C}$ NMR $(100 \mathrm{MHz}$, $\left.\mathrm{CD}_{3} \mathrm{CN}\right) \delta 152.4,138.6,138.0,136.4,132.6,129.7\left(\mathrm{C}_{\text {arom }}\right), 68.8\left(\mathrm{CH}_{2} \mathrm{Ph}\right), 51.2,50.4,48.3,48.2,48.2,48.1,47.9\left(\mathrm{CH}_{2}\right)$, 31.6, $31.0\left(\mathrm{C}\left({ }^{\mathrm{t}} \mathrm{Bu}\right)\right)$, 30.3, $29.9\left(\mathrm{Me}\left({ }^{ } \mathrm{Bu}\right)\right)$; HRMS (ES) expected for $\mathrm{C}_{27} \mathrm{H}_{47} \mathrm{~N}_{4} \mathrm{OZn}$ : 507.3036, found 507.3036. 


\section{Results and Discussion}

\section{Pendent arm synthesis}

Pendent arms incorporating a protected phenol and an alkyl bromide were required to form the desired ligand complexes as outlined in Figure 1. Bromomethyl phenol derivatives were targeted for subsequent tethering to glyoxal bridged cyclam (bisaminal). 2-(Bromomethyl)-4,6-di-tert-butylphenol, 1, was synthesised using a modified literature method reported by Wieghardt and co-workers. ${ }^{54}$ Methyl-protected di-tert-butyl benzyl bromide (8) was synthesised in three steps. The methyl protecting group was introduced using basic media in conjunction with methyl iodide. The aldehyde was then reduced using $\mathrm{NaBH}_{4}$ to form the alcohol (5), which was then brominated using $\mathrm{PBr}_{3}$ in $\mathrm{CHCl}_{3}$ to give 8 . There are limited procedures available for cleaving methyl ethers, ${ }^{55}$ and as a result benzyl ethers, which are easier to cleave, were also synthesised.
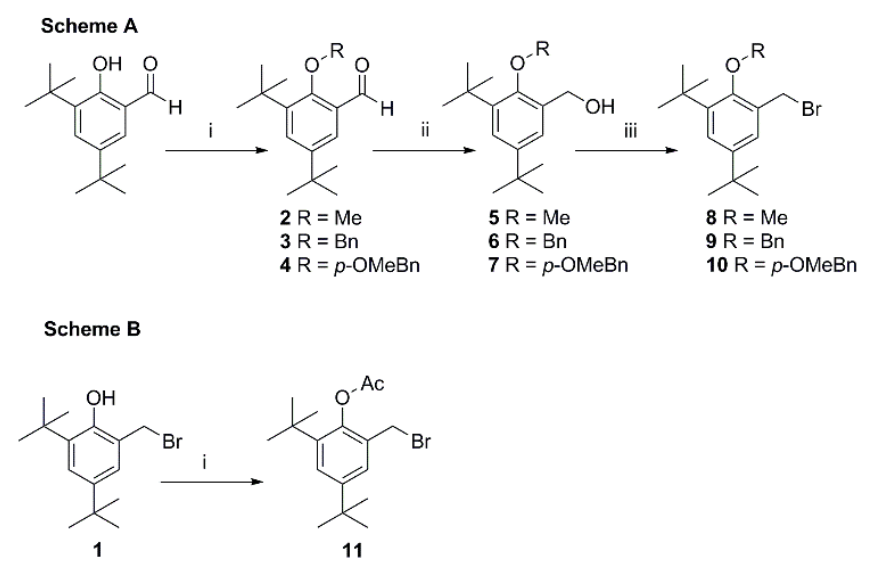

Figure 2. Synthesis of pendent arms; Scheme A : i) acetic anhydride, cat. $\mathrm{H}_{2} \mathrm{SO}_{4}$, RT $12 \mathrm{hr}$; ii) $\mathrm{K}_{2} \mathrm{CO}_{3}$, $\mathrm{RX}(\mathrm{X}=\mathrm{I}$ or $\mathrm{Br}$ ), RT to $75^{\circ} \mathrm{C}, 15-24 \mathrm{hr}$; iii) $\mathrm{NaBH}_{4}, \mathrm{RT}, 1-3 \mathrm{hr}$; Scheme B : i) $\mathrm{PBr}_{3}, \mathrm{CHCl}_{3}\left(\mathrm{R}=\mathrm{Me}\right.$ or Bn), $0^{\circ} \mathrm{C} 1-2.5 \mathrm{hr}$ or $\mathrm{CH}_{2} \mathrm{Cl}_{2}$, $\mathrm{PPh}_{3}, \mathrm{CBr}_{4}(\mathrm{R}=p$-OMeBn$), \mathrm{RT}, 18 \mathrm{hr}$.

The benzyl group was introduced by reaction with 2,4-di-tert-butyl-2-hydroxy benzaldehyde in the presence of potassium carbonate using DMF to give 3 as the product. A similar type of reaction has been reported by Counsell and co-workers in the synthesis of di-substituted 1-phenol-2-propanones, ${ }^{56}$ and by Belmar and Jiménez for preparing hindered polyanionic chelating ligands. ${ }^{57}$ Crystals of $\mathbf{3}$ were grown from hexane by evaporation; the structure elucidated is shown in Figure 3. The benzene rings in each molecule are rotated perpendicular to one another. This is extended to the asymmetric unit where benzene rings opposite each other are again found to be in a perpendicular arrangement. A similar procedure to the formation of $\mathbf{8}$ was then used to reduce the aldehyde to form the alcohol which was again brominated using $\mathrm{PBr}_{3}$ to give 9 .

Synthesis of $\mathbf{1 0}$ was carried out in an analogous manner to the synthesis of $\mathbf{9}$ apart from the bromination step. $p$ Methoxybenzyl cleavage can occur under acidic conditions and so carbon tetrabromide/ $\mathrm{PPh}_{3}$ were used to produce $\mathbf{1 3}$ in $68 \%$ yield after column chromatography. 


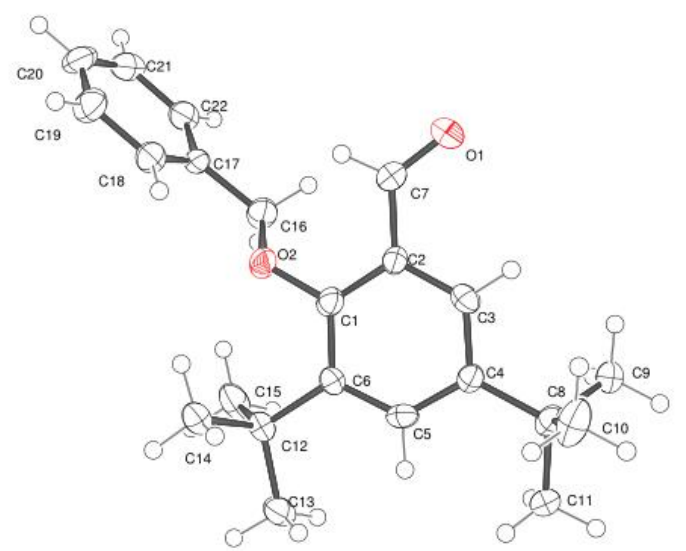

Figure 3. ORTEP representation (50\% thermal ellipsoids) of the X-ray crystal structure of $\mathbf{3}$ with all non-H atoms labelled

The synthesis of 11 was initially attempted using a dimethylaminopyridine (DMAP) coupling between 2,4,-di-tert-butyl2-hydroxy benzaldehyde and $\mathrm{Boc}_{2} \mathrm{O} .{ }^{58}$ The reaction was judged complete after $3 \mathrm{hr}$ by $\mathrm{TLC}\left(R_{f}=0.32\right.$ in $5 \%$ EtOAc/hexane compared with 0.59 for the starting material). However, reduction of the aldehyde using $\mathrm{NaBH}_{4}$ also reduced the ester, reforming the phenol. The acetyl protected phenol compound was instead synthesised from $\mathbf{1}$. Wieghardt and co-workers have protected 6-tert-butyl-o-cresol as the acetyl ester using acetic anhydride at RT with $\mathrm{H}_{2} \mathrm{SO}_{4}$ as a catalyst. ${ }^{59}$ Using the same conditions, 1 was efficiently protected using these conditions and, after an aqueous work-up, 11 was obtained in $>95 \%$ yield. Crystals of $\mathbf{1 1}$ suitable for X-ray crystallography were grown by evaporation of a toluene solution; the structure is shown in Figure 4.

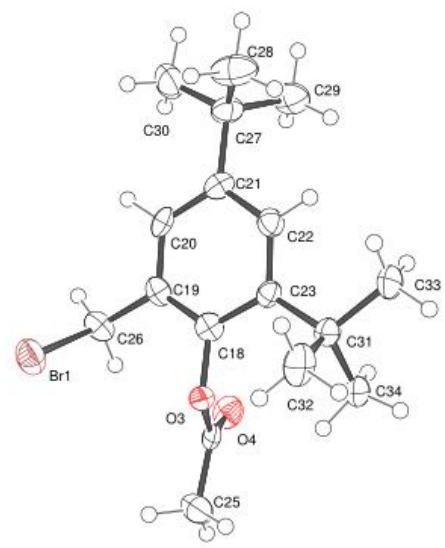

Figure 4. ORTEP representation (50\% thermal ellipsoids) of the X-ray crystal structure of $\mathbf{1 1}$ with all non-H atoms labelled.

\section{Functionalised azamacrocycle synthesis}

An initial attempt was made to attach 1, to the bisaminal cyclam bridged with glyoxal (12) ${ }^{60}$ Upon the addition of dry MeCN to a mixture of $\mathbf{1}$ and 12, an immediate precipitate formed which was analysed by NMR and mass spectrometry. Analyses provided evidence for $\mathbf{1 2}$ acting as a non-nucleophilic base; the precipitate was the hydrobromide salt of cyclam. Focus was, therefore, diverted to the use of the protected phenolate pendent arms.

Reaction of the methyl ether derivative, $\mathbf{8}$, with $\mathbf{1 2}$ gave the desired mono-alkylated product. This bis-aminal species 
was then reduced using $\mathrm{NaBH}_{4}$ in ethanol to form the piperazine ring, giving the side-bridged cyclam component, $\mathbf{L}^{2}$. $\mathbf{L}^{3}, \mathbf{L}^{\mathbf{4}}$ and 17 were synthesised in an analogous way.

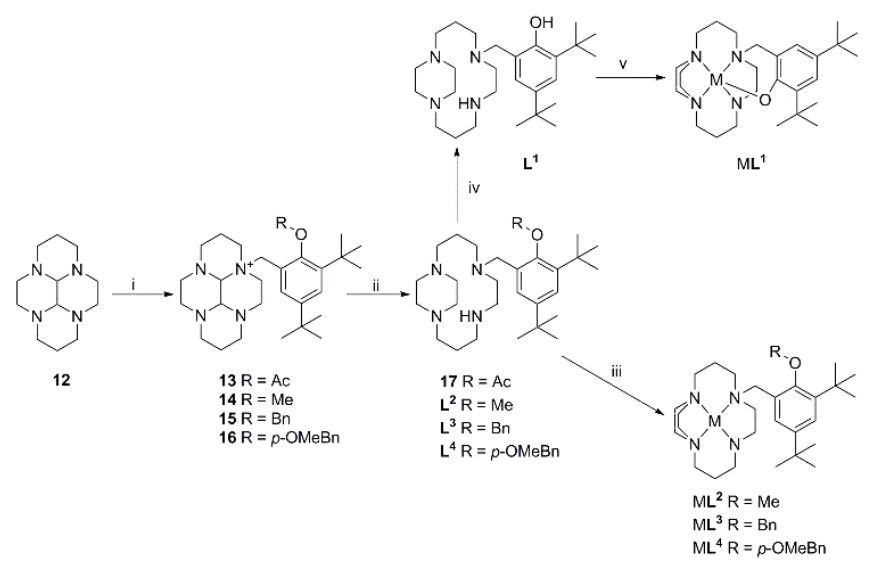

Figure 5. Synthesis of the macrocyclic ligands and their resulting metal complexes $(\mathrm{M}=\mathrm{Ni}, \mathrm{Cu}$ or $\mathrm{Zn})$; i) $\mathbf{8 , 9}, \mathbf{1 0}$ or $\mathbf{1 1}$, MeCN, RT, 3 days; ii) $\mathrm{NaBH}_{4}$, ethanol or methanol, 2 - 7 days, RT; iii) $\left[\mathrm{M}\left(\mathrm{ClO}_{4}\right)_{2}\right]$, $\mathrm{MeOH}, 2 \mathrm{hr}$, reflux; iv) $6 \mathrm{M} \mathrm{HCl}$, $24 \mathrm{hr}$, reflux; v) $\mathrm{NEt}_{3},\left[\mathrm{M}\left(\mathrm{ClO}_{4}\right)_{2}\right], \mathrm{MeOH}, 2 \mathrm{hr}$, reflux.

$\mathrm{BBr}_{3}$ was used in an attempt to unmask the phenol ${ }^{61}$ of $\mathbf{L}^{\mathbf{2}}$, under a series of conditions. The temperature was varied between $-80^{\circ} \mathrm{C}$ and $\mathrm{RT}$, and the reaction time was varied, between 2,4 and $12 \mathrm{hr}$. A subsequent reaction using the borontrifluoride ethyl etherate and sodium iodide reagent system ${ }^{62}$ was also attempted. Neither route was successful, and this was attibuted to the steric hindrance around the ether group in $\mathbf{L}^{2}$.

The deprotection of $\mathbf{L}^{3}$ proved similarly challenging, even though there are a number of methologies for the removal of benzyl protecting groups. ${ }^{55}$ Catalytic hydrogenolysis could not be successfully employed as both $N$-benzylic and $O$ benzylic moieties are present, even though conditions similar to those reported for cleaving a benzyl group used to protect 2,4-di-tert-butyl phenol linked to an aromatic amine by an amide bond were employed. ${ }^{57}$

A light-initiated process was also attempted. The rapid debenzylation of sterically hindered benzyl ethers is reported by Binkley and Hehemann, ${ }^{63}$ which has been further developed by Riley and Grindley. ${ }^{64}$ However, the conditions described did not result in the desired product being isolated. A series of other strategies were also attempted. Boron trifluoride ethyl etherate in conjunction with $\mathrm{NaI},{ }^{62}$ Amberlyst- $15,{ }^{65}$ ferric chloride, ${ }^{66,67}$ and acetyl bromide ${ }^{68}$ in alcoholic media were all utilised but none of these routes afforded the desired ligand, $\mathbf{L}^{\mathbf{1}}$.

The $p$-methoxybenzyl derivatives offered alternative reactions for the deprotection step. The synthesis of the protected species was again via the bis-aminal intermediate. A variety of methods were attempted including acetic acid at a range of temperatures ${ }^{69}$ and cerium chloride hydrate/ sodium iodide. ${ }^{70}$ However, $\mathbf{L}^{\mathbf{1}}$ was again not obtained using these deprotection strategies. Hampton and Harmata report that they too were unable to unmask a 2,4-di-tert-butyl moiety in their PHZ (5,6,11,12-tetrahydro-2,8-dimethylphenhomazine) based ligands, so the results reported here for $\mathbf{L}^{\mathbf{3}}$ and $\mathbf{L}^{\mathbf{4}}$ are consistent in this regard. ${ }^{71}$

17 was synthesised via our established route and in contrast to the previously synthesised derivatives, the acetyl group could be successfully removed to unmask the phenol. $6 \mathrm{M} \mathrm{HCl}$ at reflux was found to be sufficient to cleave the acetyl protecting group to afford $\mathbf{L}^{\mathbf{1}}$.

\section{Complex formation}

The $\mathrm{Zn}(\mathrm{II}), \mathrm{Cu}(\mathrm{II})$ and $\mathrm{Ni}(\mathrm{II})$ complexes of the phenolic ethers, $\mathbf{L}^{\mathbf{2}}, \mathbf{L}^{\mathbf{3}}$ and $\mathbf{L}^{\mathbf{4}}$, were synthesised and purified by size exclusion chromatography on a Sephadex LH-20 column. Yields for the transition metal complexes of $\mathbf{L}^{2}, \mathbf{L}^{3}$ and $\mathbf{L}^{4}$ 
were in the range of $95-98 \%, 42-87 \%$ and $29-49 \%$ respectively.

Orange crystals of the nickel(II) complex of $\mathbf{L}^{2}$ suitable for X-ray diffraction were grown by evaporating an acetoneether solution at RT. The oxygen atom from the methoxy group of the pendent arm is not utilised in the coordination to the nickel(II) centre, which adopts a distorted square planar geometry with the four nitrogen atoms of the macrocyclic ring (bond lengths for the nickel(II) ion are given in Table 1). There is disorder in the tert-butyl group para to the methoxy group, with each atom occupying two sites $\sim 50 \%$ of the time (only one site shown in Figure 6). This structure contrasts with the $\mathrm{Ni}(\mathrm{II})$ cyclam complex bearing a pendent phenolate arm reported by Iitaka $e$ t $a l^{21}$, in which the $\mathrm{Ni}(\mathrm{II})$ centre is octahedral. The oxygen of the phenolate fills one apical site with a Ni-O bond length of $2.015 \AA$. This distance is shorter than the four Ni-N bond lengths $(2.072,2.051,2.085$ and $2.078 \AA$ ) and far shorter than apical bond le ngths in octahedral nickel(II)-cyclam complexes ${ }^{72,73}\left(2.492 \AA\right.$ for $\mathrm{X}=\mathrm{Cl}, 2.169 \AA$ for $\left.\mathrm{X}=\mathrm{NO}_{3}\right)$. The other apical site is filled by an oxygen of a perchlorate anion, which has a bond length of $2.402 \AA$. The rigidity of the side-bridged cyclam in $\left[\mathrm{NiL}^{2}\right]^{2+}$ results in a contraction of the Ni-N bond length by $\sim 0.1 \AA$. The cyclam ligand adopts a trans-II configuration which is conistent with all other piperazino methyl cyclam-based chelators. ${ }^{50}$ To our knowledge, $\left[\mathrm{NiL}^{2}\right]^{2+}$, is the first reported side-bridged cyclam based species with a central $\mathrm{Ni}(\mathrm{II})$ ion.

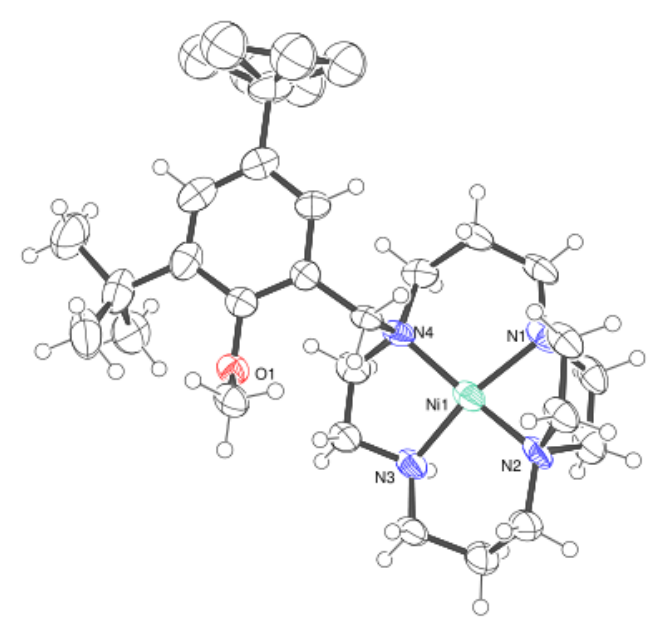

Figure 6. ORTEP representation (50\% thermal ellipsoids) of the X-ray crystal structure of $\left[\mathrm{NiL}^{2}\right]^{2+}$ with all non-H atoms labelled. Water molecules and perchlorate anions have been removed for clarity

Table 1. Selected bond lengths from the X-ray crystal structure of $\left[\mathrm{NiL}^{2}\right]\left(\mathrm{ClO}_{4}\right)_{2}$.

\begin{tabular}{lc}
\hline & Bond length $(\AA)$ \\
\hline $\mathrm{Ni}(1)-\mathrm{N}(1)$ & $1.941(5)$ \\
$\mathrm{Ni}(1)-\mathrm{N}(2)$ & $1.922(6)$ \\
$\mathrm{Ni}(1)-\mathrm{N}(3)$ & $1.921(5)$ \\
$\mathrm{Ni}(1)-\mathrm{N}(4)$ & $1.953(5)$ \\
\hline
\end{tabular}

Complexation reactions of $\mathbf{L}^{\mathbf{1}}$ to $\mathrm{Cu}(\mathrm{II}), \mathrm{Ni}(\mathrm{II})$ and $\mathrm{Zn}$ (II) were performed in basic media to ensure deprotonation of the phenol. These complexes are very hygroscopic, and were isolated as oils in yields ranging between $29-40 \%$. The ${ }^{1} \mathrm{H}$ NMR spectrum of $\left[\mathrm{ZnL}^{1}\right]^{+}$possessed the furthest downfield chemical shifts for the two aromatic protons in the phenol ring compared to the analogous complexes $\mathbf{L}^{2}-\mathbf{L}^{4}$. 


\section{UV-Vis spectroscopy}

UV-Vis data for the $\mathrm{Cu}(\mathrm{II})$ and $\mathrm{Ni}(\mathrm{II})$ complexes of $\mathbf{L}^{\mathbf{1}}-\mathbf{L}^{\mathbf{4}}$ are presented in table 2. The electronic spectrum of $\left[\mathrm{NiL}^{2}\right]^{2+}$ would suggest that the ion is square planar as a single $d$ - $d$ transition was observed at $477 \mathrm{~nm}$. Lindoy and co-workers ${ }^{74}$ observed only one band for their $N$-benzylated cyclam complexes in the region of 474-488 nm, all of which were square planar. $\left[\mathrm{CuL}^{2}\right]^{2+}$ also possesses one $d-d$ band at $547 \mathrm{~nm}$ in the $\mathrm{UV}$, which is diagnostic of a copper(II)- $\mathrm{N}_{4}$ chromophore, in which solvent or anion may occupy axial positions. ${ }^{75}$ However, the featureless nature of the spectrum results in it being of little use for the assignment of a detailed coordination geometry around the copper(II) ion.

Table 2. Tabulated UV-Vis data for the $\mathrm{Cu}(\mathrm{II})$ and $\mathrm{Ni}(\mathrm{II})$ complexes of $\mathbf{L}^{\mathbf{1}}-\mathbf{L}^{\mathbf{4}}$

\begin{tabular}{|l|l|l|}
\hline Complex & $\lambda_{\max } / \mathrm{nm}$ & $\varepsilon / \mathrm{mol}^{-1} \mathrm{dm}^{3} \mathrm{~cm}^{-1}$ \\
\hline$\left[\mathrm{CuL}^{1}\right]\left(\mathrm{ClO}_{4}\right)$ & 641 & 27 \\
\hline$\left[\mathrm{CuL}^{2}\right]\left(\mathrm{ClO}_{4}\right)_{2}$ & 547 & 331 \\
\hline$\left[\mathrm{CuL}^{3}\right]\left(\mathrm{ClO}_{4}\right)_{2}$ & 550 & 286 \\
\hline$\left[\mathrm{CuL}^{4}\right]\left(\mathrm{ClO}_{4}\right)_{2}$ & 529 & 154 \\
\hline$\left[\mathrm{NiL}^{1}\right]\left(\mathrm{ClO}_{4}\right)$ & 441 & 12 \\
\hline$\left[\mathrm{NiL}^{2}\right]\left(\mathrm{ClO}_{4}\right)_{2}$ & 477 & 197 \\
\hline$\left[\mathrm{NiL}^{3}\right]\left(\mathrm{ClO}_{4}\right)_{2}$ & & 100 \\
\hline$\left[\mathrm{NiL}^{4}\right]\left(\mathrm{ClO}_{4}\right)_{2}$ & 481 & 96 \\
\hline
\end{tabular}

Electronic spectra of $\left[\mathrm{CuL}^{3}\right]^{2+}$ and $\left[\mathrm{NiL}^{3}\right]^{2+}$ were similar to the analogous complexes formed with $\mathbf{L}^{2}$. Two single $d$ - $d$ bands were observed at 550 and $481 \mathrm{~nm}$ for the copper(II) and nickel(II) complexes respectively. [ $\left.\mathrm{NiL}^{3}\right]^{2+}$ is, therefore, square planar whereas it is again difficult to ambiguously assign the coordination around the copper(II) ion in $\left[\mathrm{CuL}^{3}\right]^{2+}$. Similar electronic spectra were observed for the copper(II) and nickel(II) complexes of $\mathbf{L}^{4}$; two single $d$ - $d$ bands were again observed at 529 and $478 \mathrm{~nm}$ for the copper(II) and nickel(II) complexes respectively. The presence of the different phenolic ethers produced would, therefore, appear to have little effect on the coordination sphere around the metal centre.

However, the electronic spectrum of $\left[\mathrm{CuL}^{1}\right]^{+}$displayed a single $d-d$ transition at $641 \mathrm{~nm}$. The shift observed is indicative of a five-coordinate geometry (either distorted square based pyramidal or trigonal bipyramidal) being formed around the metal ion. ${ }^{76}$ This would imply that the phenolate ligand occupies the apical site in solution. The electronic spectrum of $\left[\mathrm{NiL}_{1}\right]^{+}$possesses one strong $d-d$ transition at $441 \mathrm{~nm}$ and a weaker one at $581 \mathrm{~nm}$. In the nickel(II) complex of a phenolate cyclam ligand produced by Kimura and co-workers ${ }^{40}$ different absorptions are observed dependent on $\mathrm{pH}$. At acidic and basic $\mathrm{pH}$, absorptions at 424 and $559 \mathrm{~nm}$ were observed respectively, corresponding to a transition from low spin to high spin. ${ }^{77}$ This data suggests that in the nickel(II) complex of $\mathbf{L}^{1}$, the nickel(II) ion adopts either a square planar geometry with the phenol not bound to the metal centre, or an octahedral geometry in which the phenol is bound to the metal in the axial site and a solvent molecule occupies the remaining site. 


\section{Cyclic voltammetry}

Cyclic voltammetry was conducted to probe the redox behaviour of the $\mathrm{Cu}(\mathrm{II})$ and $\mathrm{Ni}(\mathrm{II})$ complexes of $\mathbf{L}^{1}-\mathbf{L}^{4}$ and the ease that the phenolate moiety of $\mathbf{L}^{1}$ could be oxidised to form a phenoxyl radical.

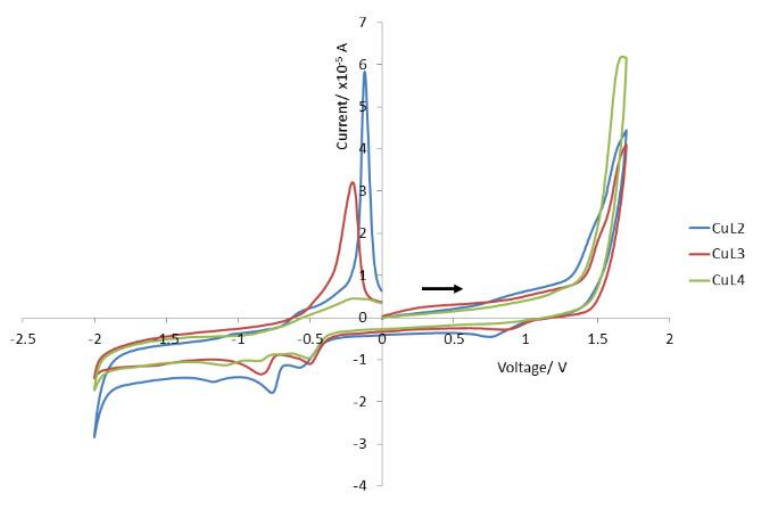

Figure 7. Cyclic voltammograms of $\left[\mathrm{CuL}^{2}\right]^{2+},\left[\mathrm{CuL}^{3}\right]^{2+}$ and $\left[\mathrm{CuL}^{4}\right]^{2+}$ at $0.2 \mathrm{~V} / \mathrm{s}$. Data collected in acetonitrile with tetrabutylammonium perchlorate as a supporting electrolyte at $\mathrm{RT}$ using a $\mathrm{Ag} / \mathrm{AgCl}$ reference electrode.

Figure 7 shows the cyclic voltammograms for $\left[\mathrm{CuL}^{2}\right]^{2+},\left[\mathrm{CuL}^{3}\right]^{2+}$ and $\left[\mathrm{CuL}^{4}\right]^{2+}$. The cyclic voltammograms for $\left[\mathrm{CuL}^{2}\right]^{2+}$, $\left[\mathrm{CuL}^{3}\right]^{2+}$ and $\left[\mathrm{CuL}^{4}\right]^{2+}$ compare well with those obtained by Camus et. al. ${ }^{78}$ who studied $C$ - and $N$-functionalised cyclams to tune the coordination properties of the chelated copper(II). The irreversible copper(II) reduction to copper(I) is evidenced for all three complexes by the presence a wave at $\sim-0.8 \mathrm{~V}$; the peak at $\sim-0.5 \mathrm{~V}$ is due to the same process for metallic copper. An oxidation peak at $\sim-0.15 \mathrm{~V}$, characteristic of copper(0) to copper(II), is also observed. This irreversible peak, which is due to anodic stripping caused by the redissolution of metallic copper, is indicative of copper(0) formation due to dismutation of dissociated copper(II) ions. ${ }^{79}$ Furthermore, this suggests that the electrogenerated copper(I) formed is unstable and dissociates during the timescale of the electrochemical experiment. The anodic stripping process appears to be sensitive to the alkyl group at the phenolic site, in that the current intensity for this process is greatest for $\left[\mathrm{CuL}^{2}\right]^{2+}$ and weakest for $\left[\mathrm{CuL}^{4}\right]^{2+}$ i.e. $\left[\mathrm{CuL}^{2}\right]^{2+}>\left[\mathrm{CuL}^{3}\right]^{2+}>\left[\mathrm{CuL}^{4}\right]^{2+}$

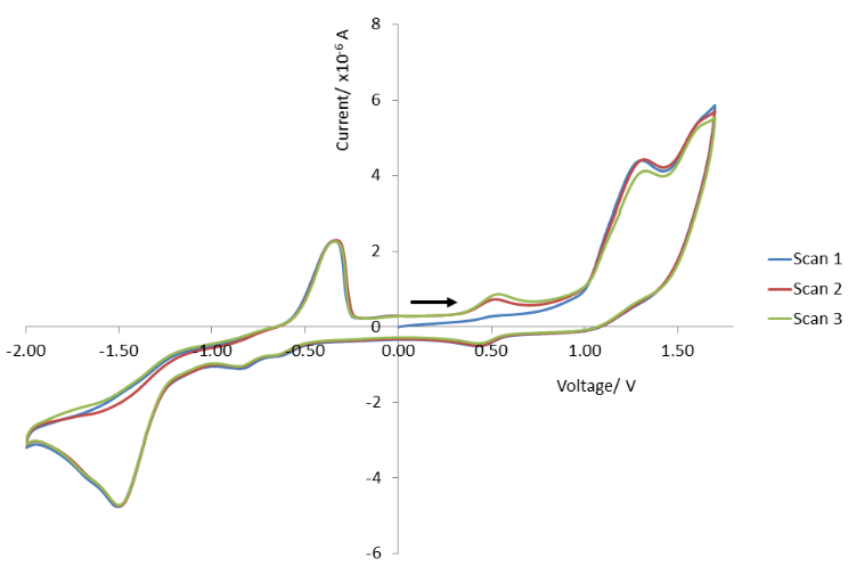

Figure 8. Consecutive cyclic voltammograms of $\left[\mathrm{CuL}^{1}\right]^{+}$at $0.2 \mathrm{~V} / \mathrm{s}$. Data collected in acetonitrile with tetrabutylammonium perchlorate as a supporting electrolyte at $\mathrm{RT}$ using a $\mathrm{Ag} / \mathrm{AgCl}$ reference electrode.

Figure 8 shows the cyclic voltammagram of $\left[\mathrm{CuL}^{1}\right]^{+}$. At $+0.48 \mathrm{~V}$ a fully reversible peak is observed, which is assigned 
to the formation of the phenoxyl radical and subsequent reduction back to the phenolate. Reversibility of this wave was tested by plotting the peak current verses the square root of the scan rate. The resulting plot was linear; indicative of a reversible process. Previous work by Wieghardt and co-workers has been focused on the study of numerous metal complexes with phenolate pendent arms. ${ }^{54}$ Their studies have shown that the redox potential for the phenoxyl radical/phenolate couple can vary depending on the metal centre. For a TACN (triazacyclononane) zinc(II) complex with a di-tert-butyl phenolate and two methyl pendent arms, the redox potential for the reversible formation of the phenoxyl radical is between $-0.09 \mathrm{~V}$ and $-0.32 \mathrm{~V}$. When the methyl pendent arms are exchanged for acetates, values of $0.63,0.73$ and $0.36 \mathrm{~V}$ are obtained for the gallium(III), iron(III) and cobalt(III) metal complexes respectively. ${ }^{22}$ One of us has also reported a cyclam-based ligand system analogous to $\mathbf{L}^{1}$ (in terms of the pendent arm) that displays a single quasireversible peak at $-0.58 \mathrm{~V}$ in the cyclic voltammogram. ${ }^{80}$ The irreversible peak at $+1.27 \mathrm{~V}$ is due to oxidation of an $\mathrm{OH}$ group, implying that the phenolate ligand has been protonated, and as such is not coordinated to the copper(II) centre. The difference in peak intensity between this peak and that of the phenoxyl radical suggests that the former is dominant. The appearance of the anodic region of the cyclic voltammaogram is very similar to that of those 7-, 8- and 9-hydroxy3-ethoxycarbonyl-2,4-dimethyl coumarin[4,3-b] pyridine isomers studied by Pardo-Jiménez et al. ${ }^{81}$

The copper-based electrochemistry of $\left[\mathrm{Cu}^{1}\right]^{+}$is very similar to that of $\left[\mathrm{Cu} \mathbf{L}^{2}\right]^{2+},\left[\mathrm{Cu}^{3}\right]^{2+}$ and $\left[\mathrm{Cu} \mathbf{L}^{4}\right]^{2+}$. In the cathodic region the oxidation of copper(II) to copper(I) is again observed for the complex as well as metallic copper. Furthermore, anodic stripping is observed as an irreversible wave at $-0.45 \mathrm{~V}$.

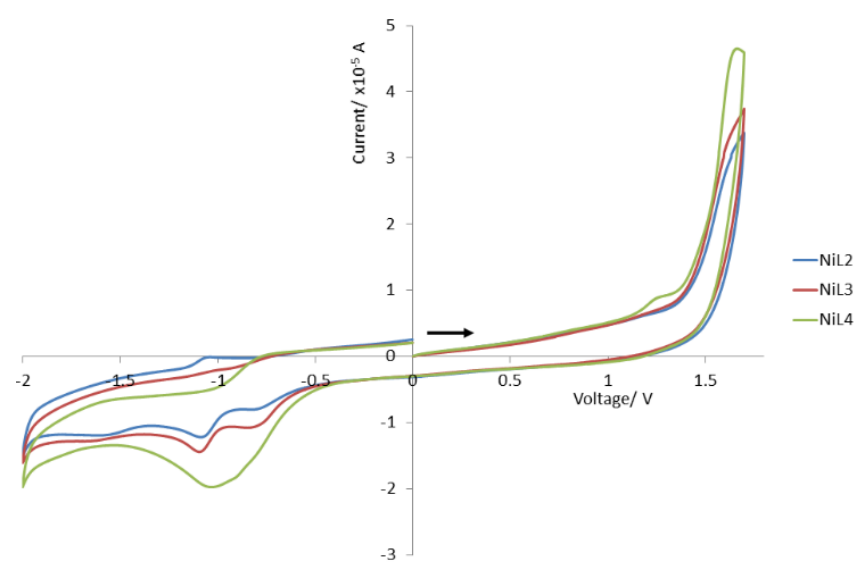

Figure 9. Cyclic voltammograms of $\left[\mathrm{Ni}^{2}\right]^{2+},\left[\mathrm{NiL}^{3}\right]^{2+}$ and $\left[\mathrm{NiL}^{4}\right]^{2+}$ recorded at $0.2 \mathrm{~V} / \mathrm{s}$. Data collected in acetonitrile with tetrabutylammonium perchlorate as a supporting electrolyte at $\mathrm{RT}$ using a $\mathrm{Ag} / \mathrm{AgCl}$ reference electrode.

Figure 9 shows the cyclic voltammograms of the three nickel(II) complexes of $\mathbf{L}^{\mathbf{2}}, \mathbf{L}^{\mathbf{3}}$ and $\mathbf{L}^{\mathbf{4}}$. The cyclic voltammogram of $\left[\mathrm{NiL}^{4}\right]^{2+}$ differs considerably from $\left[\mathrm{Ni}^{2}\right]^{2+}$ and $\left[\mathrm{NiL}^{3}\right]^{2+}$, in that the nickel(II) to nickel(I) reduction for complexed and metallic nickel appears as a broad wave rather than two discrete waves in the cathodic region. In addition, it also displays a small irreversible oxidation wave at $+1.22 \mathrm{~V}$ which is tentatively assigned to the $\mathrm{Ni}$ (II)/ $\mathrm{Ni}$ (III) redox couple. Literature values for this process from other cyclam complexes vary between +1.07 and $+1.52 \mathrm{~V},{ }^{74}$ and +1.07 to +1.16 $\mathrm{V} .{ }^{77}\left[\mathrm{NiL}^{2}\right]^{2+}$ is the only complex to show some reversibility of the nickel(II) to nickel(I) reduction (wave at $-1.1 \mathrm{~V}$ ). 


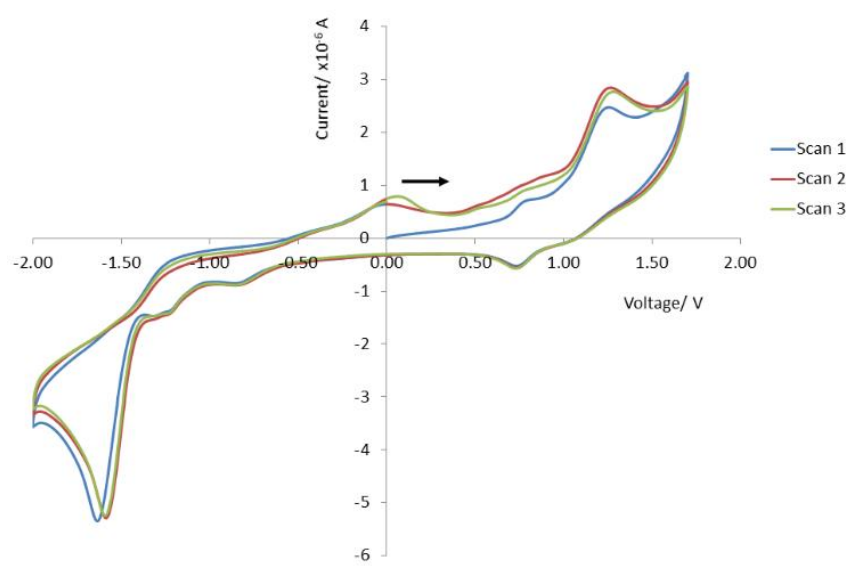

Figure 10. Consecutive cyclic voltammograms of $\left[\mathrm{Ni}_{\mathbf{1}}\right]^{+} 0.2 \mathrm{~V} / \mathrm{s}$. Data collected in acetonitrile with tetra-butyl ammonium perchlorate as a supporting electrolyte at $\mathrm{RT}$ using a $\mathrm{Ag} / \mathrm{AgCl}$ reference electrode.

Figure 10 shows the cyclic voltammograms collected at a scan rate of $0.2 \mathrm{~V} / \mathrm{s}$ for $\left[\mathrm{NiL}^{\mathbf{1}}\right]^{+}$. The reversible formation of the phenoxyl radical is observed at $+0.74 \mathrm{~V}$ (reversibility of this wave was tested by plotting peak current verses the square root of scan rate; again a linear relationship ws observed). This is significantly more positive than for the copper(II) complex, meaning that the nickel(II) ion must be stabilising this redox process to a greater extent (i.e. a more positive potential is required to generate the radical species). Kimura and co-workers reported that the phenolate undergoes oxidation at $+0.9 \mathrm{~V}$ at $\mathrm{pH} 10$ for their nickel(II) complex of a cyclam ligand possessing a phenolate compared to $+0.5 \mathrm{~V}$ uncoordinated. ${ }^{21}$ The oxidative peak at $+1.24 \mathrm{~V}$ is again due to the oxidation of the $\mathrm{OH}$; this irreversible reduction is only $0.03 \mathrm{~V}$ different to the same electrochemical process when observed for $\left[\mathrm{Cu} \mathbf{L}^{1}\right]^{+}$.

An interesting feature of the cyclic voltammogram arises in the second and third scans in that the initial oxidation of the phenolate to the phenoxyl radical is no longer clearly defined. Previously, nickel(II) complexes have been observed to possess two sets of quasi-reversible waves which are both part of the Ni(II)/Ni(III) process. ${ }^{74,77}$ The relative size of these pairs of waves can vary considerably with the nature of the ligand, solvent and electrode. Oxidation potentials for the second set of waves occur between $\sim+0.5$ to $+1 \mathrm{~V}$. It is, therefore, possible that a second set of waves for the $\mathrm{Ni}(\mathrm{II}) / \mathrm{Ni}$ (III) couple is formed after the initial scan near to the reversible wave of the phenoxyl radical. This may well lead to the distortion of the peak shape of the latter wave to the slope that is observed in scans two and three.

\section{Conclusions}

Side-bridged cyclam transition based metal complexes $(\mathrm{M}=\mathrm{Ni}(\mathrm{II}), \mathrm{Cu}(\mathrm{II})$ and $\mathrm{Zn}(\mathrm{II}))$ bearing a phenolic ether or a phenolate pendent arm have been synthesised and characterised. UV-vis studies revealed that the identity of the phenolic ether had no effect on the geometry of the chelated metal ion. However, $\left[\mathrm{CuL}^{\mathbf{1}}\right]^{+}$and $\left[\mathrm{NiL}^{\mathbf{1}}\right]^{+}$showed significant differences in that the former was square-based pyramidal whilst the $\mathrm{Ni}$ (II) ion in the latter existed in either a squareplanar or an octaedral geometry. For the $\mathrm{Ni}(\mathrm{II})$ and $\mathrm{Cu}(\mathrm{II})$ complexes of $\mathbf{L}^{\mathbf{1}}$, evidence for a stable phenoxyl radical species is obtained (reversible peaks at $+0.74 \mathrm{~V}$ and $+0.48 \mathrm{~V}$ respectively), although evidence for the oxidation of $\mathrm{OH}$, due to protonation of phenolate, is observed for both complexes at $\sim+1.24 \mathrm{~V}$. The phenoxyl radical in $\left[\mathrm{Ni}\left(\mathbf{L}^{\mathbf{1}}\right)\right]^{2+}$ complex is much harder to oxidise by $0.26 \mathrm{~V}$ compared to the analogous $\mathrm{Cu}$ (II) complex. The $\mathrm{Ni}$ (II) ion in the crystal structure of $\left[\mathrm{Ni}\left(\mathbf{L}^{\mathbf{1}}\right)\right]\left[\left(\mathrm{ClO}_{4}\right)_{2}\right]$ possesses a distorted square-planar geometry in which the phenolic ether pendent arm is not involved 
in the coordination sphere. The cyclam ligand adopts a trans-II configuration. To our knowledge, this represents the first reported structural characterisation of a $\mathrm{Ni}(\mathrm{II})$ side-bridged cyclam complex.

Supporting Information CCDC 906943-909645 contain the supplementary crystallographic data for $\mathbf{3}, \mathbf{1 1}$ and $\left[\mathrm{NiL}^{2}\right]^{2+}$. These data can be obtained free of charge from The Cambridge Crystallographic Data Centre via www.ccdc.cam.ac.uk/data_request/cif.

Acknowledgments REM wishes to thank the University of Hull for a studentship. SJA would like to thank the Daisy Appeal charity (grant no. DAhul2011) and for funding, and Dr Assem Allam and his family for the generous donation to help found the PET Research Centre at the University of Hull and for their continued support. We acknowledge the EPSRC UK National Mass Spectrometry Facility at Swansea University for the collection of mass spectrometry data.

\section{Declarations of interest: none}




\section{References}

(1) Boswell, C. A.; Regino, C. A. S.; Baidoo, K. E.; Wong, K. J.; Bumb, A.; Xu, H.; Milenic, D. E.; Kelley, J. A.; Lai, C. C.; Brechbiel, M. W. Bioconjugate Chem. 2008, 19, 1476-1484.

(2) Boswell, C. A.; Sun, X.; Niu, W.; Weisman, G. R.; Wong, E. H.; Rheingold, A. L.; Anderson, C. J. J. Med. Chem. 2004, 47, 1465-1474.

(3) Burke, B. P.; Clemente, G. S.; Archibald, S. J. J. Labelled Comp. Radiopharm. 2014, 57, 239-243.

(4) Dale, A. V.; Il An, G.; Pandya, D. N.; Ha, Y. S.; Bhatt, N.; Soni, N.; Lee, H.; Ahn, H.; Sarkar, S.; Lee, W.; Huynh, P. T.; Kim, J. Y.; Gwon, M.-R.; Kirn, S. H.; Park, J. G.; Yoon, Y.-R.; Yoo, J. Inorg. Chem. 2015, 54, 8177-8186.

(5) Aung, W.; Jin, Z.-H.; Furukawa, T.; Claron, M.; Boturyn, D.; Sogawa, C.; Tsuji, A. B.; Wakizaka, H.; Fukumura, T.; Fujibayashi, Y.; Dumy, P.; Saga, T. Molecular imaging 2013, 12, 376-387.

(6) Gomes, A. J.; Espreafico, E. M.; Tfouni, E. Mol. Pharmaceut. 2013, 10, 3544-3554.

(7) Yu, M.; Lim, N. H.; Ellis, S.; Nagase, H.; Triccas, J. A.; Rutledge, P. J.; Todd, M. H. Chemistryopen 2013, 2, 99-105.

(8) Blahut, J.; Hermann, P.; Galisova, A.; Herynek, V.; Cisarova, I.; Tosner, Z.; Kotek, J. Dalton Trans. 2016, 45, 474-478.

(9) Fisher, C. M.; Fuller, E.; Burke, B. P.; Mogilireddy, V.; Pope, S. J. A.; Sparke, A. E.; DechampsOlivier, I.; Cadiou, C.; Chuburu, F.; Faulkner, S.; Archibald, S. J. Dalton Trans. 2014, 43, 9567-9578. (10) Poty, S.; Gourni, E.; Desogere, P.; Boschetti, F.; Goze, C.; Maecke, H. R.; Denat, F. Bioconjugate Chem. 2016, 27, 752-761.

(11) Boros, E.; Holland, J. P.; Kenton, N.; Rotile, N.; Caravan, P. ChemPlusChem 2016, 81, 274-281.

(12) David, T.; Kubicek, V.; Gutten, O.; Lubal, P.; Kotek, J.; Pietzsch, H.-J.; Rulisek, L.; Hermann, P. Inorg. Chem. 2015, 54, 11751-11766.

(13) Halime, Z.; Frindel, M.; Camus, N.; Orain, P.-Y.; Lacombe, M.; Cherel, M.; Gestin, J.-F.; FaivreChauvet, A.; Tripier, R. Org. Bio. Chem. 2015, 13, 11302-11314.

(14) Burke, B. P.; Seemann, J.; Archibald, S. J. In Advances in Inorganic Chemistry, Vol 68: Insights from Imaging in Bioinorganic Chemistry, VanEldik, R.; Hubbard, C. D., Eds., 2016, pp 301-339.

(15) Liang, X.; Parkinson, J. A.; Weishäupl, M.; Gould, R. O.; Paisey, S. J.; Park, H.-s.; Hunter, T. M.; Blindauer, C. A.; Parsons, S.; Sadler, P. J. J. Am. Chem. Soc. 2002, 124, 9105-9112.

(16) Khan, A.; Silversides, J. D.; Madden, L.; Greenman, J.; Archibald, S. J. Chem. Commun. 2006, 416-418.

(17) Fricker, S. P. Transfus. Med. Hemother. 2013, 40, 237-245.

(18) Ross, A.; Choi, J.-H.; Hunter, T. M.; Pannecouque, C.; Moggach, S. A.; Parsons, S.; De Clercq, E.; Sadler, P. J. Dalton Trans. 2012, 41, 6408-6418.

(19) Poty, S.; Desogere, P.; Goze, C.; Boschetti, F.; D'Huys, T.; Schols, D.; Cawthorne, C.; Archibald, S. J.; Maecke, H. R.; Denat, F. Dalton Trans. 2015, 44, 5004-5016.

(20) Maples, R. D.; Cain, A. N.; Burke, B. P.; Silversides, J. D.; Mewis, R. E.; D'Huys, T.; Schols, D.; Linder, D. P.; Archibald, S. J.; Hubin, T. J. Chem. Eur. J. 2016, 22, 12916-12930.

(21) Iitaka, Y.; Koike, T.; Kimura, E. Inorg. Chem. 1986, 25, 402-404.

(22) Kimura, S.; Bill, E.; Bothe, E.; Weyhermüller, T.; Wieghardt, K. J. Am. Chem. Soc. 2001, 123, 6025-6039.

(23) Mewis, R. E.; Archibald, S. J. Coord. Chem. Rev. 2010, 254, 1686-1712.

(24) Chen, H.-H.; Yang, J.; Liu, Y.-Y.; Ma, J.-F. CrystalEngComm 2013, 15, 5168-5178.

(25) Matz, D. L.; Jones, D. G.; Roewe, K. D.; Gorbet, M.-J.; Zhang, Z.; Chen, Z.; Prior, T. J.;

Archibald, S. J.; Yin, G.; Hubin, T. J. Dalton Trans. 2015, 44, 12210-12224.

(26) Wilson, K. R.; Cannon-Smith, D. J.; Burke, B. P.; Birdsong, O. C.; Archibald, S. J.; Hubin, T. J. Polyhedron 2016, 114, 118-127.

(27) Murray, B. S.; New, E. J.; Pal, R.; Parker, D. Org. Bio. Chem. 2008, 6, 2085-2094.

(28) Lau, Y. H.; Clegg, J. K.; Price, J. R.; Macquart, R. B.; Todd, M. H.; Rutledge, P. J. Chem. Eur. J. 2018, 24, 1573-1585.

(29) Wong, J. K. H.; Todd, M. H.; Rutledge, P. J. Molecules 2017, 22.

(30) Pujols-Ayala, I.; Barry, B. A. Biochim. Biophys. Acta-Bioenerg. 2004, 1655, 205-216. 
(31) Rogers, M. S.; Dooley, D. M. Curr. Opin. Chem. Biol. 2003, 7, 189-196.

(32) Auerbach, U.; Eckert, U.; Wieghardt, K.; Nuber, B.; Weiss, J. Inorg. Chem. 1990, 29, 938-944.

(33) Kimura, S.; Bill, E.; Bothe, E.; Weyhermüller, T.; Wieghardt, K. J. Am. Chem. Soc. 2001, 123, 6025-6039.

(34) Nirmala, G.; Rahiman, A. K.; Sreedaran, S.; Jegadeesh, R.; Raaman, N.; Narayanan, V.

Polyhedron 2011, 30, 106-113.

(35) Moore, D. A.; Fanwick, P. E.; Welch, M. J. Inorg. Chem. 1989, 28, 1504-1506.

(36) Kimura, E. Pure Appl. Chem. 1986, 58, 1461-1466.

(37) Kimura, E.; Koike, T.; Takahasi, M. J. Chem. Soc., Chem. Commun. 1985, 385-386.

(38) Kimura, E.; Koike, T.; Uenishi, K.; Davidson, R. B. J. Chem. Soc., Chem. Commun. 1986, 11101111.

(39) Kimura, E.; Koike, T.; Uenishi, K.; Hediger, M.; Kuramoto, M.; Joko, S.; Arai, Y.; Kodama, M.; Iitaka, Y. Inorg. Chem. 1987, 26, 2975-2983.

(40) Kimura, E.; Uenishi, K.; Koike, T.; Iitaka, Y. Chem. Lett. 1986, 1137-1140.

(41) Maria, L.; Santos, I. C.; Alves, L. G.; Marçalo, J.; Martins, A. M. J. Organomet. Chem. 2013, 728, 57-67.

(42) Maria, L.; Santos, I. C.; Sousa, V. R.; Marçalo, J. Inorg. Chem. 2015, 54, 9115-9126.

(43) Bellouard, F.; Chuburu, F.; Kervarec, N.; Toupet, L.; Triki, S.; Le Mest, Y.; Handel, H. J. Chem. Soc., Perkin Trans. 1 1999, 3499-3505.

(44) J. Davies, P.; R. Taylor, M.; Wainwright, K. P. Chem. Commun. 1998, 827-828.

(45) Royal, G.; Dahaoui-Gindrey, V.; Dahaoui, S.; Tabard, A.; Guilard, R.; Pullumbi, P.; Lecomte, C. Eur. J. Org. Chem. 1998, 1998, 1971-1975.

(46) Le Baccon, M.; Chuburu, F.; Toupet, L.; Handel, H.; Soibinet, M.; Dechamps-Olivier, I.; Barbiere, J. P.; Aplincourt, M. New J. Chem. 2001, 25, 1168-1174.

(47) Abdulwahaab, B. H.; Burke, B. P.; Domarkas, J.; Silversides, J. D.; Prior, T. J.; Archibald, S. J. J. Org. Chem. 2016, 81, 890-898.

(48) Silversides, J. D.; Burke, B. P.; Archibald, S. J. Comptes Rendus Chimie 2013, 16, 524-530.

(49) Boswell, C. A.; Regino, C. A. S.; Baidoo, K. E.; Wong, K. J.; Milenic, D. E.; Kelley, J. A.; Lai, C. C.; Brechbiel, M. W. Biorg. Med. Chem. 2009, 17, 548-552.

(50) Smith, R.; Huskens, D.; Daelemans, D.; Mewis, R. E.; Garcia, C. D.; Cain, A. N.; Freeman, T. N. C.; Pannecouque, C.; De Clercq, E.; Schols, D.; Hubin, T. J.; Archibald, S. J. Dalton Trans. 2012, 41, 11369-11377.

(51) Sheldrick, G. M. Acta Cryst. 2008, A64, 112-122.

(52) Farrugia, L. J. J. Appl. Crystallogr. 1999, 32, 837-838.

(53) Farrugia, L. J. J. Appl. Crystallogr. 1997, 30, 565.

(54) Sokolowski, A.; Müller, J.; Weyhermüller, T.; Schnepf, R.; Hildebrandt, P.; Hildenbrand, K.;

Bothe, E.; Wieghardt, K. J. Am. Chem. Soc. 1997, 119, 8889-8900.

(55) Weissman, S. A.; Zewge, D. Tetrahedron 2005, 61, 7833-7863.

(56) Counsell, R. E.; Desai, P.; Kulkarni, P. G. J. Med. Chem. 1971, 14, 789-792.

(57) Jiménez, C. A.; Belmar, J. B. Tetrahedron 2005, 61, 3933-3938.

(58) Hansen, M. H.; Riggs, J. R. Tetrahedron Lett. 1998, 39, 2705-2706.

(59) Stockheim, C.; Hoster, L.; Weyhermüller, T.; Wieghardt, K.; Nuber, B. J. Chem. Soc., Dalton

Trans. 1996, 4409-4416.

(60) Le Baccon, M.; Chuburu, F.; Toupet, L.; Handel, H.; Soibinet, M.; Dechamps-Olivier, I.; Barbier, J.-P.; Aplincourt, M. New J. Chem. 2001, 25.

(61) McOmie, J. F. W.; West, D. E. Organic Synthetic Collection 1973, 5, 412-414.

(62) Vankar, Y. D.; Rao, C. T. J. Chem. Res., Synop . 1985, 232-233.

(63) Binkley, R. W.; Hehemann, D. G. J. Org. Chem. 1990, 55, 378-380.

(64) Riley, J. G.; Grindley, T. B. J. Carbohydr. Chem. 2001, 20, 159-169.

(65) Petchmanee, T.; Ploypradith, P.; Ruchirawat, S. J. Org. Chem. 2006, 71, 2892-2895.

(66) Rodebaugh, R.; Debenham, J. S.; Fraser-Reid, B. Tetrahedron Lett. 1996, 37, 5477-5478.

(67) Park, M. H.; Takeda, R.; Nakanishi, K. Tetrahedron Lett. 1987, 28, 3823-3824. 
(68) Lesk, A.; Nudelman, A. Synth. Commun. 1999, 29, 1405-1408.

(69) Hodgetts, K. J.; Wallace, T. W. Synth. Commun. 1994, 24, 1151-1155.

(70) Cappa, A.; Marcantoni, E.; Torregiani, E. J. Org. Chem. 1999, 64, 5696-5699.

(71) Hampton, C. S.; Harmata, M. Tetrahedron 2016, 72, 6064-6077.

(72) Bosnich, B.; Poon, C. K.; Tobe, M. L. Inorg. Chem. 1965, 4, 1102-1108.

(73) Thom, V. J.; Fox, C. C.; Boeyens, J. C. A.; Hancock, R. D. J. Am. Chem. Soc. 1984, 106, 59475955.

(74) Dong, Y.; Lawrence, G. A.; Lindoy, L. F.; Turner, P. Dalton Trans. 2003, 1567-1576.

(75) Fabbrizzi, L.; Montagna, A.; Poggi, A.; Kaden, T. A.; Siegfried, L. C. Dalton Trans. 1987, 26312634.

(76) Silversides, J. D.; Allan, C. C.; Archibald, S. J. Dalton Trans. 2007, 971-978.

(77) Dong, Y.; Lindoy, L. F.; Turner, P.; Wei, G. Dalton Trans. 2004, 1264-1270.

(78) Camus, N.; Le Bris, N.; Nuryyeva, S.; Chessé, M.; Esteban-Gómez, D.; Platas-Iglesias, C.; Tripier, R.; Elhabiri, M. Dalton Trans. 2017, 46, 11479-11490.

(79) Grujicic, D.; Pesic, B. Electrochim. Acta 2002, 47, 2901-2912.

(80) Aranburu Leiva, A.; Kaur, M.; Benjamin, S.; Jones, A.; Langley, S.; Mewis, R. Molbank 2017, 2017, M963.

(81) Pardo-Jiménez, V.; Barrientos, C.; Squella, J. A.; Navarrete-Encina, P. A.; Nuñez-Vergara, L. J. J. Electrochem. Soc. 2011, 158, F166-F172. 